\title{
Comparison of geosynchronous energetic particle flux responses to solar wind dynamic pressure enhancements and substorms
}

\author{
D.-Y. Lee \\ Department of Astronomy and Space Science, College of Natural Sciences and Institute for Basic Science Research, \\ Chungbuk National University, Chungbuk, South Korea \\ L. R. Lyons \\ Department of Atmospheric Sciences, University of California, Los Angeles, California, USA \\ G. D. Reeves \\ Space Science and Applications, Los Alamos National Laboratory, Los Alamos, New Mexico, USA \\ Received 18 February 2005; revised 25 April 2005; accepted 3 May 2005; published 9 September 2005.
}

[1] Geosynchronous energetic particle fluxes are used to examine the differences and similarities between the particle disturbances due to an enhancement in solar wind dynamic pressure $P_{\mathrm{dyn}}$ and those caused by substorms. Disturbances are also distinguished by IMF conditions. First, for not strongly southward IMF conditions (weakly southward or northward IMF), we find that the magnetospheric compression by a $P_{\text {dyn }}$ enhancement usually causes particle fluxes to increase simultaneously at all energy channels. The increase is global around the Earth, but it usually occurs first on the dayside and then propagates to the nightside within a few minutes. We also find that a magnetospheric compression sometimes leads to a flux decrease or no flux change for at least one energy channel at some MLTs, which we attribute to the shape of radial profiles at constant adiabatic invariants. However, we find no evidence for substorm-like injections in our $P_{\mathrm{dyn}}$ enhancement events when the IMF is not strongly southward. Following prolonged strongly southward IMF, substorms caused by IMF changes that lead to convection electric field reduction and are not associated with a $P_{\text {dyn }}$ change generate flux disturbances that are quite similar to typical substorm flux disturbances for less strongly southward IMF conditions. However, the dispersionless injection front is found over a much wider azimuthal region, sometimes extending to the late afternoonside for protons. We find that under prolonged steady, strongly southward IMF conditions, a $P_{\text {dyn }}$ enhancement leads to a two-mode type disturbance. The disturbance due to magnetospheric compression can be clearly identified and is seen primarily on the dayside, and a substorm-like injection associated with current wedge formation is seen on the nightside. The dayside compression effect is seen in both species, but is often more easily identified in the proton fluxes than in the electron fluxes. The substorm-like injection feature is also seen in both species but is usually more evident in the electron fluxes. In the events studied here, the dayside compression disturbance precedes the substorm-like injection on the nightside by a few minutes.

Citation: Lee, D.-Y., L. R. Lyons, and G. D. Reeves (2005), Comparison of geosynchronous energetic particle flux responses to solar wind dynamic pressure enhancements and substorms, J. Geophys. Res., 110, A09213, doi:10.1029/2005JA011091.

\section{Introduction}

[2] It has long been known from numerous observations that the flux of the energetic particles in the near-Earth magnetosphere often changes significantly. In particular the geosynchronous and near tail regions are places where these changes are easily observed in association with substorms and other geomagnetic disturbances. Typically, substorms

Copyright 2005 by the American Geophysical Union. 0148-0227/05/2005JA011091\$09.00 create particle flux enhancements at energies of tens to hundreds of $\mathrm{keV}$, which are often preceded by flux decreases. The enhancement is dispersionless in energy within an injection region near midnight MLT but is dispersed at MLTs away from these regions [e.g., Reeves, 1998; Birn et al., 2000]. (See also Figure 1, for example, which will be further discussed below.)

[3] A significant magnetospheric particle flux disturbance can also be caused by a sudden change in solar wind conditions. For example, Li et al. [2003] suggested that a solar wind dynamic pressure enhancement associated with 


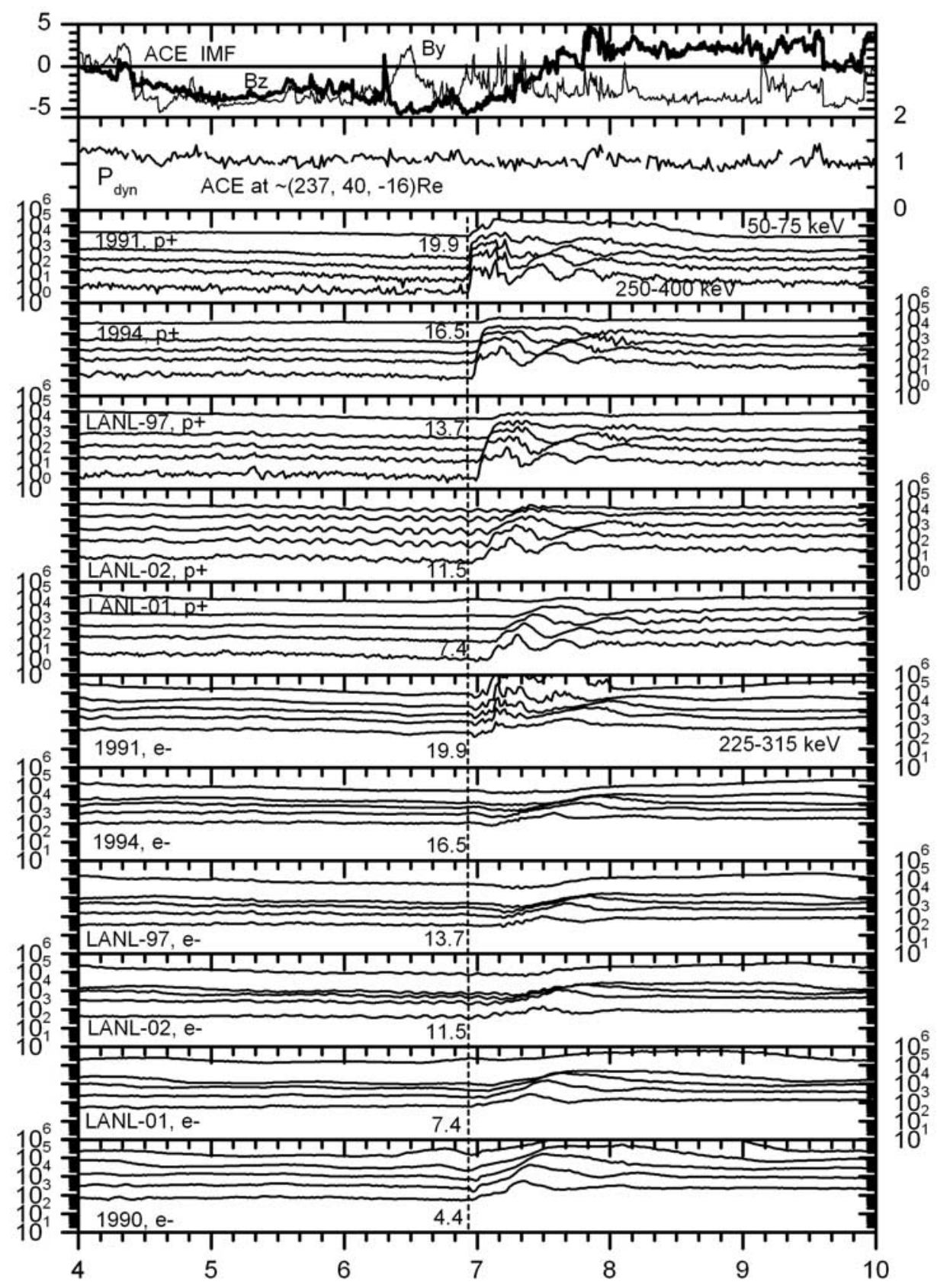

May 12, 2002

Figure 1. IMF $B_{y}, B_{z}$ (in $\mathrm{nT}$ ), and $P_{\text {dyn }}($ in $\mathrm{nPa}$ ) as observed by the ACE spacecraft (upper two panels), and flux (number $/ \mathrm{cm}^{2} \mathrm{~s}$ sr $\mathrm{keV}$ ) of energetic protons (as indicated by $\mathrm{p}+$ ) and electrons (as indicated by e-) from the several LANL geosynchronous spacecraft for a substorm on 12 May 2002. The time of flux disturbance initiation is indicated by a vertical dashed line. The MLT values of each LANL spacecraft at this time are indicated near the vertical line. The five energy channels are $50-75 \mathrm{keV}$ through $250-400 \mathrm{keV}$ for protons, and 50-70 keV through 225-315 keV for electrons.

an interplanetary shock can induce significant flux changes at geosynchronous orbit, both on the dayside and the nightside. Recently, Lee et al. [2004] reported four sawtooth events where the quasi-periodic variations in energetic particle flux are due to series of continuous solar wind dynamic pressure enhancements.
[4] There are many aspects of the disturbances of the magnetosphere-ionosphere system caused by solar-wind dynamic pressure, $P_{\text {dyn }}$. These include a ground magnetic field disturbance [e.g., Russell et al., 1994, and references therein], a geosynchronous magnetic field response [e.g., Wing et al., 2002; Lee and Lyons, 2004, and references 
therein], a response throughout the magnetotail [e.g., Nakai et al., 1991; Kawano et al., 1992; Fairfield and Jones, 1996; Collier et al., 1998; Ostapenko and Maltsev, 1998; Kim et al., 2004], an auroral disturbance [e.g., Lyons et al., 2000; Zesta et al., 2000; Chua et al., 2001; Boudouridis et al., 2003], and a polar cap convection change [Lukianova, 2003; Boudouridis et al., 2004]. To our knowledge, however, there is no published comprehensive study of how the response of energetic particle flux within the inner magnetosphere to a solar wind dynamic pressure change compares with substorm energetic particle injections.

[5] The primary purpose of the present work is to understand the basic nature of the response of the energetic particle flux to dynamic pressure enhancements using the data obtained from geosynchronous spacecraft. We are particularly interested in how the response is different from or similar to typical substorm injections and how sensitive the flux disturbance is to different IMF conditions.

[6] In Paper 1 [Lyons et al., 2005] we perform a similar examination of the auroral disturbance due to dynamic pressure enhancement using global auroral images from the WIC imager on the IMAGE spacecraft. In that paper, we consider first the substorm and dynamic pressure responses under not-strongly southward IMF conditions, which we refer to as "typical substorms" and "typical dynamic pressure disturbances." We then consider separately the responses under strongly southward IMF conditions and identify important differences from the typical dynamic pressure disturbances. We use the same organization in the present paper. In section 2, a brief description is given of the data used and the analysis method employed. The results of the analysis are presented in sections 3 and 4, first for substorms and dynamic pressure enhancement events where the accompanied IMF is weakly southward or northward, and then for substorms and dynamic pressure enhancement events where the associated IMF is strongly southward. Discussion and summary are given in section 5 .

\section{Data and Analysis Method}

[7] We use the geosynchronous energetic particle flux data obtained from the Los Alamos National Laboratory (LANL) geosynchronous spacecraft measurements. Mostly, data from five energy channels were used, covering the energy ranges of 50-400 keV for protons and $50-315 \mathrm{keV}$ for electrons, respectively. We use $1 \mathrm{~min}$ time resolution for all LANL data. To complement the analysis, we also use geosynchronous magnetic field data from the GOES spacecraft and geomagnetic $\mathrm{H}$ data from various low-latitude ground stations when necessary, all at $1 \mathrm{~min}$ time resolution. The availability of IMAGE WIC aurora data was also checked to complement the work in Paper 1 (i.e., for completeness from a viewpoint of the IMAGE WIC study in Paper 1).

[8] We first present differences between substorm injections and the flux disturbances due to dynamic pressure enhancements for the case where the accompanied IMF is northward or only weakly southward. Next we present the substorm injections caused by IMF changes following prolonged strongly southward IMF but associated with no significant change in the solar wind dynamic pressure. Then this case is compared with the flux disturbance due to dynamic pressure enhancements when the IMF is steady and strongly southward. The solar wind and IMF conditions are determined using data from the spacecraft ACE, GEOTAIL, and WIND. In brief, we place all our examined events into four categories: substorm injection triggered by IMF changes under NOT strongly southward IMF conditions, dynamic pressure enhancement event under NOT strongly southward IMF conditions, substorm injection triggered by sole IMF changes following prolonged strongly southward IMF, and dynamic pressure enhancement event under steady and strongly southward IMF conditions.

\section{Not Strongly Southward IMF Conditions}

[9] In this section we compare the substorm injection and the flux disturbance due to the dynamic pressure enhancement when the associated IMF is weakly southward or northward.

\section{1. (Typical) Substorm Injections}

[10] Figure 1 shows an example of typical substormgenerated particle flux changes that are measured by several LANL spacecraft. Interplanetary data is available only from ACE for this event and are shown in the top two panels. In all figures in this paper, the IMF and solar wind data are presented without a time shift. In the case of ACE, a transit time of $\sim 30 \mathrm{~min}$ to an hour from the spacecraft to the Earth should be taken into account. The ACE data show that this substorm was associated with a weakly southward IMF and that there was no significant change in the dynamic pressure $P_{\text {dyn }}$. While precise mapping of IMF features from ACE to the Earth should not be expected, the ACE data show that an IMF $B_{y}$ reduction near 0620 UT was possibly the cause of this substorm [Lyons et al., 1997].

[11] The third to seventh panels of Figure 1 show the proton flux data in five energy channels from five LANL spacecraft, and the electron flux data from six LANL spacecraft are shown in the bottom six panels (One of the LANL spacecraft, 1990-095, does not provide the proton data). The major flux change starts at $\sim 0657 \mathrm{UT}$, as indicated by a vertical line. The MLT value of each spacecraft location at this time is indicated near the vertical line. First, it is seen that the flux of both species on the nightside ( 19.9 MLT) shows a dispersionless increase. This is followed by drift echoes, which result from injected particles that have magnetic drifted completely around the Earth. (Typical dispersionless increases occur within a limited MLT sector in the nightside, which is often called the injection region or front, and is where energetic particles are moved earthward onto new drift paths by the inductive electric field associated with the substorm dipolarization.) For this particular example, the dispersionless electron flux increase initiates at few minutes later (at $\sim 0700$ UT) than does the dispersionless flux increase for protons, which is typical for dispersionless injections observed a few hours before midnight [Birn et al., 1997]. Elsewhere the flux is seen to still increase but delayed in time and dispersed in energy. It is seen that the delay and the amount of dispersion increases westward for protons and eastward for electrons, as determined by the opposite directions of each species' drift motion around the Earth. These are the wellestablished key features of the typical substorm injection. 
These have been described well in the literature [e.g., Reeves, 1998; Birn et al., 2000] and will not be further examined here.

\section{2. (Typical) Dynamic Pressure Disturbances}

[12] Here we examine the particle flux response to the $P_{\text {dyn }}$ enhancement, first when the associated IMF is continuously or at least primarily northward and then when it is weakly southward. The geosynchronous particle response to pressure enhancements has been commonly observed and is well-known to those who have studied the geosynchronous environment but has not been well-documented in the published literature.

\subsubsection{Northward IMF Case}

[13] This is the case where the IMF $B_{z}$ is (nearly) continuously northward for at least $30 \mathrm{~min}$ before, at the time of, and for some time even after a dynamic pressure enhancement. An example is shown in Figure 2, where the top two panels show that the dynamic pressure sharply increased by a factor of $\sim 5$ at $\sim 1500$ UT while the IMF $B_{z}$ remained mostly northward, though close to zero, at and prior to the $P_{\mathrm{dyn}}$ enhancement. The $P_{\mathrm{dyn}}$ enhancement lasted for about $21 / 2$ hours as marked by the horizontal dotted arrowed-line. The third panel shows the geosynchronous magnetic field intensity from two GOES spacecraft. Magnetic field increases by about $27 \mathrm{nT}$ at 10.7 MLT and by about $12 \mathrm{nT}$ at 6.7 MLT are clearly seen at $\sim 1540$ UT in response to the enhanced pressure impact. The increase at 10.7 MLT is mostly due to the increase in $B_{z}$ while the other two components remain nearly unchanged. At 6.7 MLT, the three components of the magnetic field started to change from about $(45,38,92)$ to about $(52,42,100) \mathrm{nT}$. The particle flux in response to this $P_{\mathrm{dyn}}$ enhancement is shown in the rest of the panels in the same format as in Figure 1. Nearly simultaneous with the magnetic field compression, the particle flux shows enhancements at all three spacecraft locations and at most energy channels. (The highest energy electron flux at 22.6 MLT actually decreases, as discussed further below.) The three spacecraft are located at 4.7, 16.3, and 22.6 MLT at the time of the enhancement, respectively, and so the flux enhancement is global. The flux enhancement at a given spacecraft location is nearly simultaneous at all energy channels; in other words, dispersionless. The flux remains elevated for about $21 / 2$ hours as indicated by the short-dashed vertical lines, which is approximately same as the duration of the dynamic pressure enhancement. This global flux enhancement exhibits no substorm-like features in terms of dispersions and echoes and is therefore a direct compression effect, being distinguished from the typical substorm injection. A careful examination of the onset time of the flux disturbance at different MLT locations indicates that the flux disturbance occurred first at the dayside MLT (namely at 16.3) and then slightly later at the nightside MLTs. (This is also so for the magnetic field response in the third panel, namely, the B started to increase first at 10.7 MLT and then a few minutes later at 6.7 MLT.) This implies a propagation of the compression effect from the dayside toward the nightside, which is not what we see for the typical substorm injections.

[14] After examining a number of events, we have found that the flux response to the $P_{\mathrm{dyn}}$ enhancement is not always an increase but sometimes shows a decrease or very little change. One of such examples is shown in Figure 3. In the event shown, the dynamic pressure increased initially by a factor of $\sim 2$ and then had further gradual increase over an extended period while the IMF was mostly northward. The observations by the GOES 8 and 10 spacecraft at 10.8 and 6.8 MLT, respectively, clearly verify the impact of this enhanced pressure at $\sim 1543$ UT. The magnetic field intensity at GOES 8 location increased from about $120 \mathrm{nT}$ to about $170 \mathrm{nT}$ rather gradually over nearly 2 hours. Similarly, the increase at the GOES 10 location is gradual and from about $105 \mathrm{nT}$ up to about $150 \mathrm{nT}$. These increases at both spacecraft are mainly due to increases in $B_{z}$. At this time, $\sim 1543$ UT, two LANL spacecraft were located at 22.6 and 20.4 MLT, respectively, and their observations show that the flux of both species decreased or changed very little in most channels.

[15] The change in the particle flux at a given energy channel due to the compressional effect of a $P_{\text {dyn }}$ enhancement must in general be determined by a combination of adiabatic acceleration and the spatial (radial) profile of the source particle distribution at constant first and second adiabatic invariants. Thus a decrease or little change in the flux in response to a $P_{\mathrm{dyn}}$ enhancement implies that the particle distribution function at the time of a $P_{\text {dyn }}$ enhancement at the location of the spacecraft decreased or was approximately constant, rather than increased, with increasing radial distance, although this is an assumption rather than a fact that is proven by the analysis in this study. The compressional effect of a $P_{\text {dyn }}$ enhancement differs from that of substorm injections in that $P_{\text {dyn }}$ enhancements change the radial location of specific drift shells but they do not lead to localized particle "injections" and there is no drift signature which is energy-dependent.

[16] Statistically, we have selected 28 independent $P_{\text {dyn }}$ enhancement events for northward IMF from the period 1997-2000. The $P_{\text {dyn }}$ enhancements range from $61 \%$ to $900 \%$. Out of 28 events, five events are increases of less than $100 \%$, nine events are increases of 100 to $200 \%$, and 14 events are increases of larger than $200 \%$, respectively. For each of the $P_{\mathrm{dyn}}$ enhancement events, there are typically two to four LANL spacecraft available at different MLTs providing the energetic particle flux data. This leads to 70 and 78 events of flux response for protons and electrons, respectively. The numbers here are larger for electrons because one of the LANL spacecraft, 1990-095, does not provide proton data. The events for both species are relatively evenly distributed over different MLT sectors as shown in Table 1.

[17] We find that events showing decreasing flux change or no flux change are quite common. Specifically, out of 70 flux response events for protons, only 59\% show flux increase in all energy channels, while the remaining $41 \%$ show either flux decrease in at least one energy channel or no flux change in all energy channels. For electrons, out of 78 events, $54 \%$ are characterized by the increasing flux in all energy channels while $46 \%$ show either flux decrease in at least one energy channel or no flux change in all energy channels. For some of those events that show flux decrease in one or more channels, the fluxes in other channels change differently. To show the MLT distribution of the flux response events showing flux decrease or little flux 


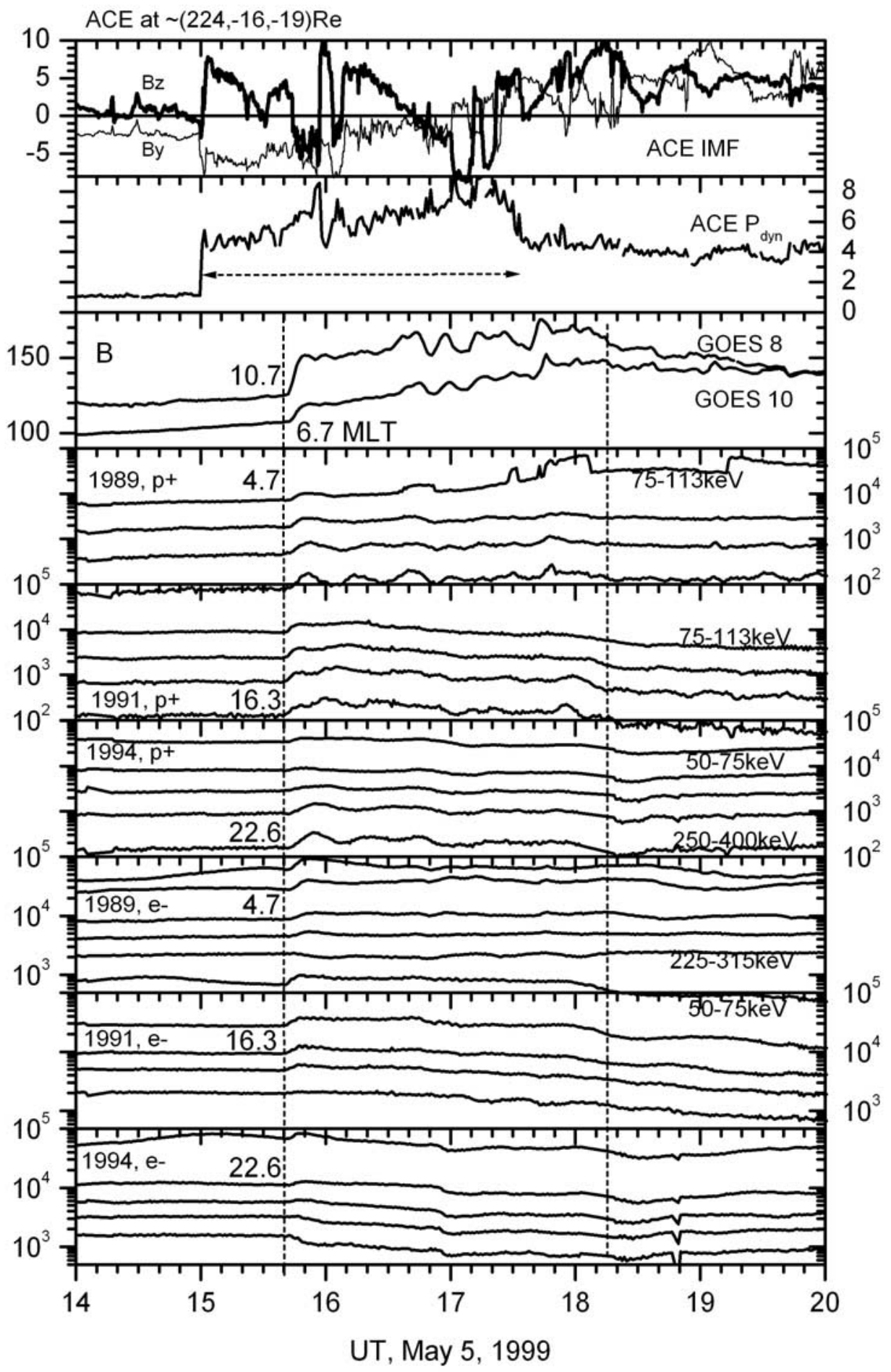

Figure 2. IMF $B_{y}, B_{z}$, and $P_{\text {dyn }}$ as observed by the ACE spacecraft (upper two panels), magnetic field in GSM from the GOES 8 and 10 geosynchronous spacecraft (third panel), and flux of energetic protons and electrons from the several LANL geosynchronous spacecraft for a $P_{\text {dyn }}$ enhancement event on 5 May 1999, presented in the same format as in Figure 1.

change, the number of such events is given in parentheses in Table 1 . It should be mentioned that a $P_{\mathrm{dyn}}$ enhancement can lead to different flux changes at different MLT regions and for different species, as well as at different energies.
[18] On the other hand, it is possible that the pattern or amount of the flux change may depend on the strength of the $P_{\text {dyn }}$ enhancement. In order to check this possibility, Figure 4 displays a stacked histogram of statistics of the number of flux response events showing flux increase in all 


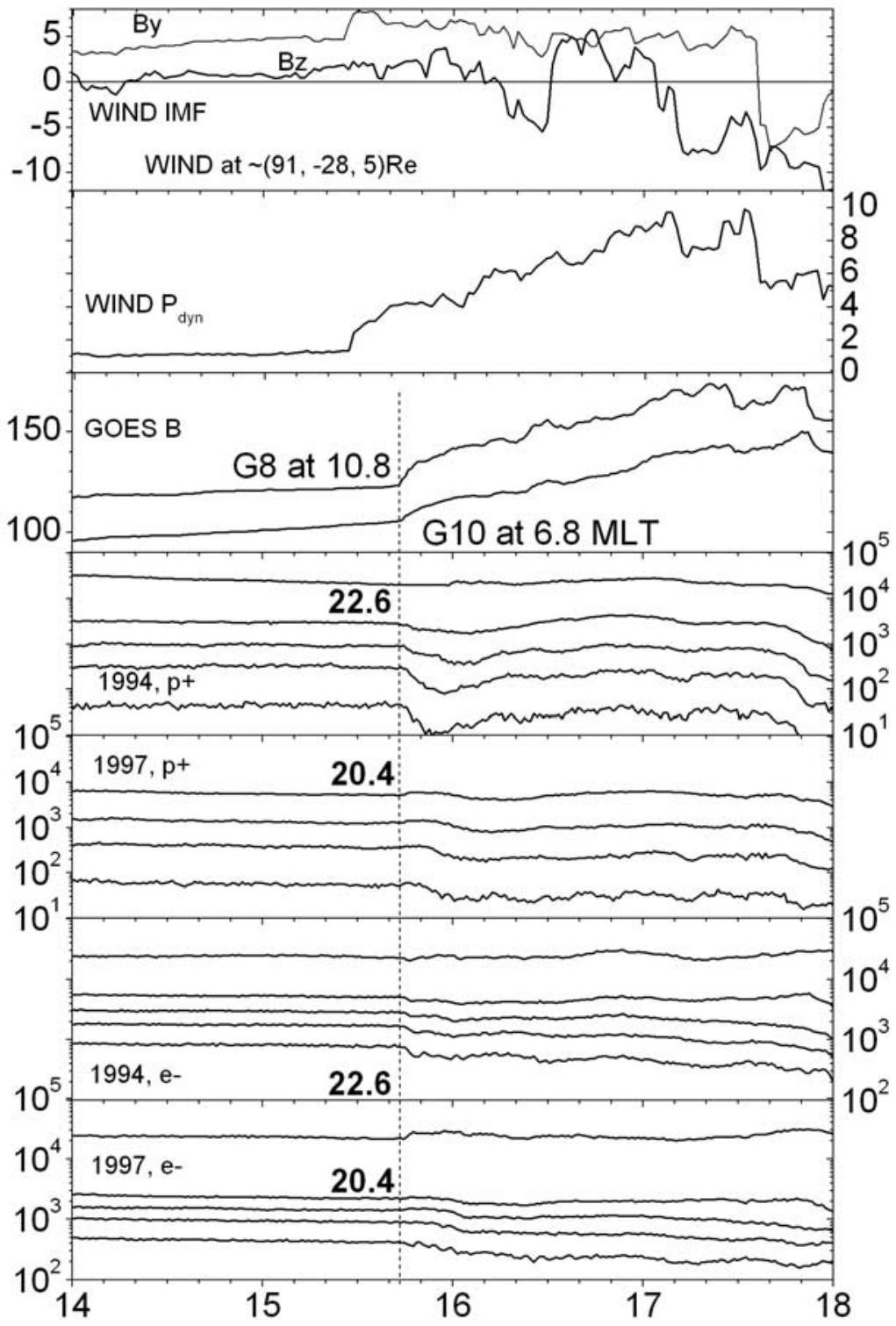

Feb. 5, 2000

Figure 3. IMF $B_{y}, B_{z}$, and $P_{\text {dyn }}$ as observed by the WIND spacecraft (upper two panels), magnetic field in GSM from the GOES 8 and 10 geosynchronous spacecraft (third panel), and flux of energetic protons and electrons from two LANL geosynchronous spacecraft for a $P_{\mathrm{dyn}}$ enhancement event on 5 February 2000 .

energy channels (dark gray bar), flux decrease in at least one energy channel (light gray bar), and no flux change in all energy channels (white blank bar) as a function of the percentage increase of the dynamic pressure. This histogram is made using the same flux response events above, namely, 70 proton events and 78 electron events (Table 1). For both protons and electrons, most of the events with no flux change are associated with relatively small $P_{\text {dyn }}$ enhancement, and the majority are for an enhancement of $100 \%$ or less. For the higher dynamic pressure enhancements, events with no flux change occur much less frequently and the flux change is primarily, but not always, an increase.

[19] For all pressure events, one needs to verify that the $P_{\text {dyn }}$ enhancement observed at the selected interplanetary
Table 1. Number of Flux Response Events in Response to 28 Independent Pressure Enhancement Events in the Present Study ${ }^{\mathrm{a}}$

\begin{tabular}{cccccc}
\hline & $0000-$ & $0600-$ & $1200-$ & $1800-$ & \\
& $0600 \mathrm{MLT}$ & $1200 \mathrm{MLT}$ & $1800 \mathrm{MLT}$ & $2400 \mathrm{MLT}$ & All MLT \\
\hline $\mathrm{p}+$ & $17(1,3)$ & $14(1,7)$ & $19(1,7)$ & $20(5,4)$ & $70(8,21)$ \\
$\mathrm{e}-$ & $18(1,5)$ & $19(1,9)$ & $19(2,7)$ & $22(6,5)$ & $78(10,26)$ \\
\hline
\end{tabular}

${ }^{a}$ The two numbers in parentheses refer to the number of events that show flux decrease in at least one energy channel and that show virtually no flux change in all energy channels, respectively. 

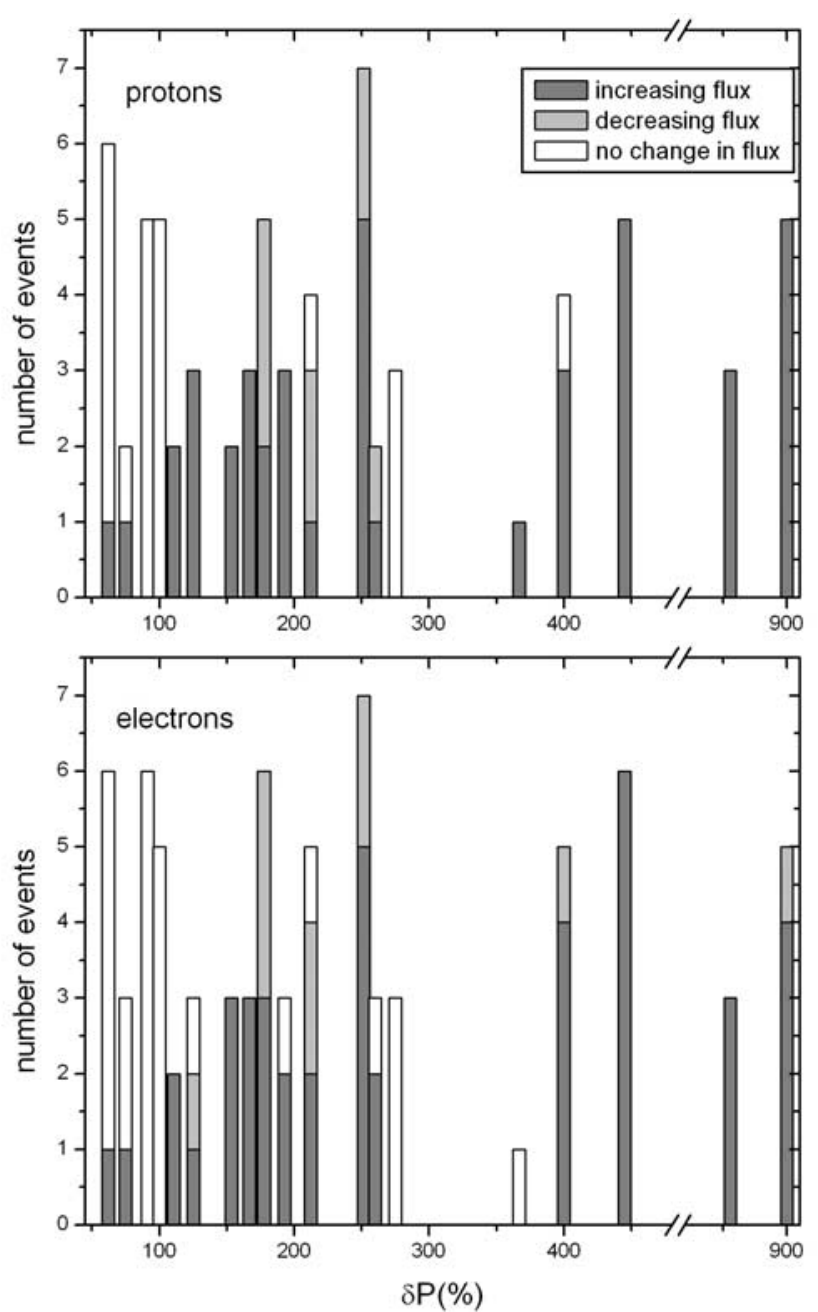

Figure 4. Statistics showing the number of events, as a function of the percentage increase in $P_{\text {dyn }}$, that the flux response to the $P_{\text {dyn }}$ enhancement is a flux increase (heavy gray), a flux decrease (light gray), and no flux change (white blank). The upper panel is for protons, the lower panel for electrons.

spacecraft does indeed represent the one that impacted the magnetosphere. This is particularly desired for the (weak to moderate) $P_{\text {dyn }}$ enhancement events that led to little flux change in a substantial number of cases. This is necessary to make sure that a small flux change is not due to spatial inhomogeneity leading to the dynamic pressure that actually impacted the magnetosphere having no enhancement although it exhibited an enhancement at the location of the selected spacecraft. We have done this by checking the lowlatitude geomagnetic $\mathrm{H}$ component for each pressure enhancement event, and we have verified that a global increase in $\mathrm{H}$, which is due to the compressional enhancement in magnetopause currents, occurred for all events studied here. In addition we have checked the geosynchronous magnetic field and have verified the compression on the dayside for all the cases where a spacecraft location was appropriate.

[20] In summary, when the accompanied IMF is northward, a $P_{\text {dyn }}$ enhancement leads to the simple compression effect, which gives either an increase, decrease, or little change in the flux, depending on the radial profile of the particle distribution function at constant adiabatic invariants. The resulting compression effect can be different for different energy channels, different MLT regions, and different particle species for a given $P_{\text {dyn }}$ enhancement. The flux response shows no evidence of substorm-type injections, which is consistent with what was found from the study using global auroral images in Paper 1.

\subsubsection{Weakly Southward IMF}

[21] When the accompanied IMF is weakly southward, we find that the response of the energetic particle flux to the $P_{\text {dyn }}$ enhancement is very similar to that of the northward IMF case. Here we present an example in Figure 5, where the top two panels display the WIND data showing that the IMF $B_{z}$ was greater than $-2 \mathrm{nT}$ at and prior to a pressure pulse at $\sim 1353$ UT. The sharp increase of the geosynchronous magnetic field by about $29 \mathrm{nT}$ at $9.8 \mathrm{MLT}$ and by about $16 \mathrm{nT}$ at 5.8 MLT is clearly seen at $\sim 1451$ UT in the third panel. At 9.8 MLT, the three components of the magnetic field changed from about $(12,35,130) \mathrm{nT}$ to about $(22,41,159) \mathrm{nT}$. At 5.8 MLT, they changed from about $(59,29,90)$ to about $(68,39,102) \mathrm{nT}$. It is seen from the rest of the panels that the basic nature of the LANL particle flux disturbance at $\sim 1451$ UT and later is practically the same as for northward IMF. Note the flux changes differ between protons and electrons, between different MLT regions, and between different energies.

[22] As the IMF becomes further southward, the flux response becomes different and much less simple. Below, we describe the major difference for strongly southward IMF conditions. However, we have not yet investigated the transition from weakly to strongly southward IMF. Also pressure pulses are often accompanied with near-simultaneous IMF turnings that might modify the pure pressure effect. These greatly limit the number of events suitable for a statistical analysis for the weakly southward IMF case.

\section{Strongly Southward IMF Conditions}

\subsection{Substorm Injections}

[23] Here we examine the particle injection characteristics caused by substorms that are triggered by IMF turnings following prolonged strongly southward IMF but associated with no significant change in dynamic pressure. Figure 6 shows an example where a substorm initiated at $\sim 0840$ UT after the IMF $B_{z}$ was $\sim-8 \mathrm{nT}$ for $\sim 30 \mathrm{~min}$ and significantly negative for at least $40 \mathrm{~min}$ before that. This substorm seems to have been triggered by the northward turning of the IMF $B_{z}$ indicated by the dotted vertical line in the second panel. The dynamic pressure, on the other hand, remained nearly steady during this time interval. The typical magnetic field dipolarization near midnight is clearly seen by the GOES measurement in the third panel. At the time of the substorm onset, three LANL spacecraft were available at 21.7, 15.6, and 13.4 MLTs, respectively. At 21.7 MLT, the flux of both species shows the typical dispersionless increase followed by weak drift echoes. It is most interesting that a similar dispersionless increase in the proton flux is seen at 15.6 MLT. This is slightly delayed by a few minutes compared with that at 21.7 MLT. The proton flux also increases at 13.4 MLT, but with a short additional delay and 


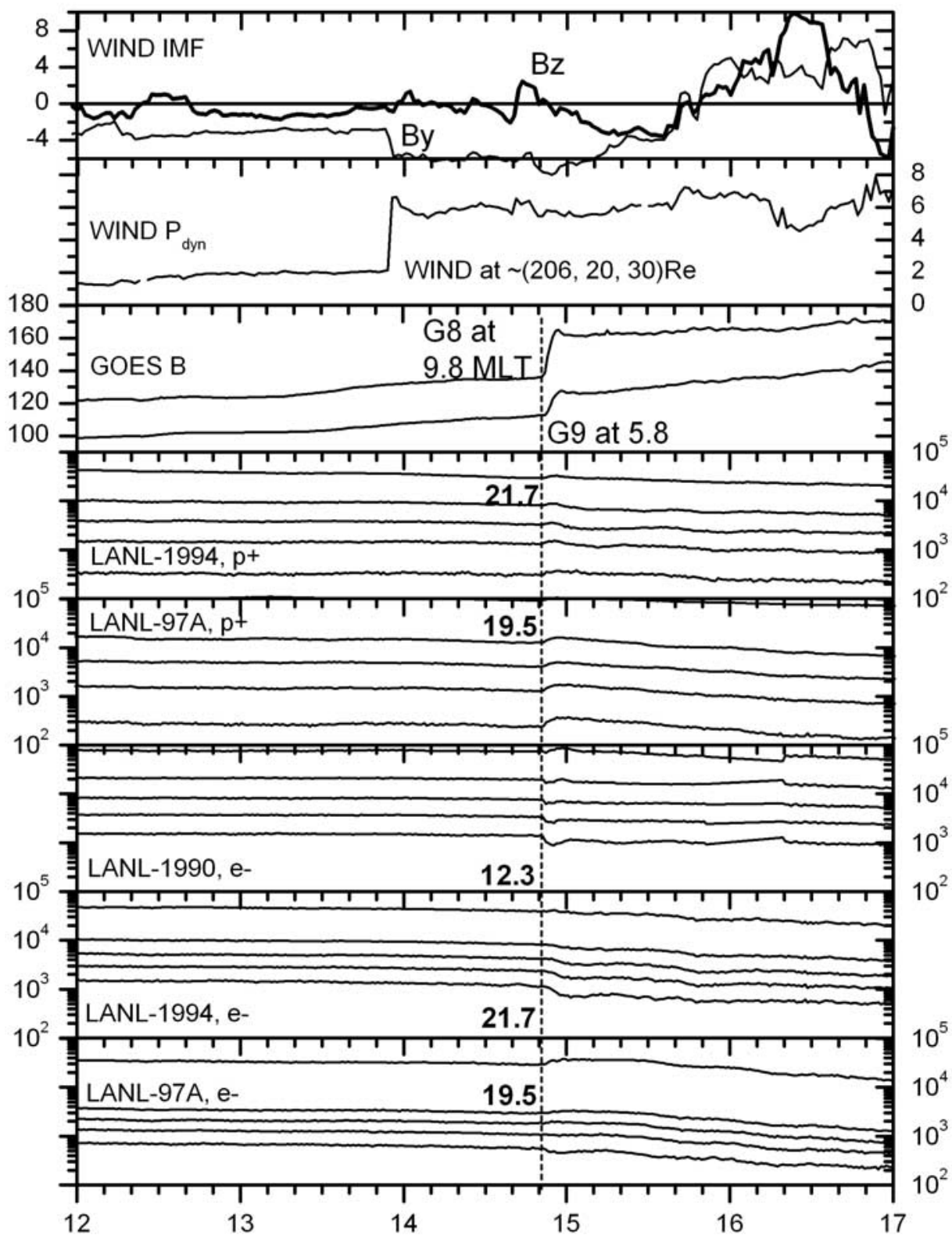

May 15, 1998

Figure 5. IMF $B_{y}, B_{z}$, and $P_{\mathrm{dyn}}$ as observed by the WIND spacecraft (upper two panels), magnetic field in GSM from the GOES 8 and 9 geosynchronous spacecraft (third panel), and flux of energetic protons and electrons from the several LANL geosynchronous spacecraft for a $P_{\text {dyn }}$ enhancement event on 15 May 1998.

some dispersion in energy. The amount of this dispersion is, however, quite small, implying that the responsible injection zone was quite close to this MLT sector. Both MLTs, 15.6 and 13.4, are the region where one would normally expect more significant dispersion than those seen in the present event. The electron flux, however, does not show the dispersionless flux increase at these dayside MLTs, but only the long-delayed and well-dispersed flux increase, which is the typical substorm signature for afternoon MLTs. This event suggests that at least the proton flux injection front can extend to some MLT sector on the afternoonside, perhaps due to azimuthal expansion of the current wedge from the nightside or radially inward propagation of a planar and already-wide injection front into geosynchronous orbit.

[24] Two other events shown in Figures 7 and 9 demonstrate the same feature. First, in Figure 7, two substorm events are identified as indicated by vertical lines. Both seem to be associated with the reduction of IMF $B_{y}$ component magnitude. The second one at $\sim 0850$ UT is more useful in demonstrating the particle injection feature. Associated with this substorm, the IMF $B_{z}$ was very 


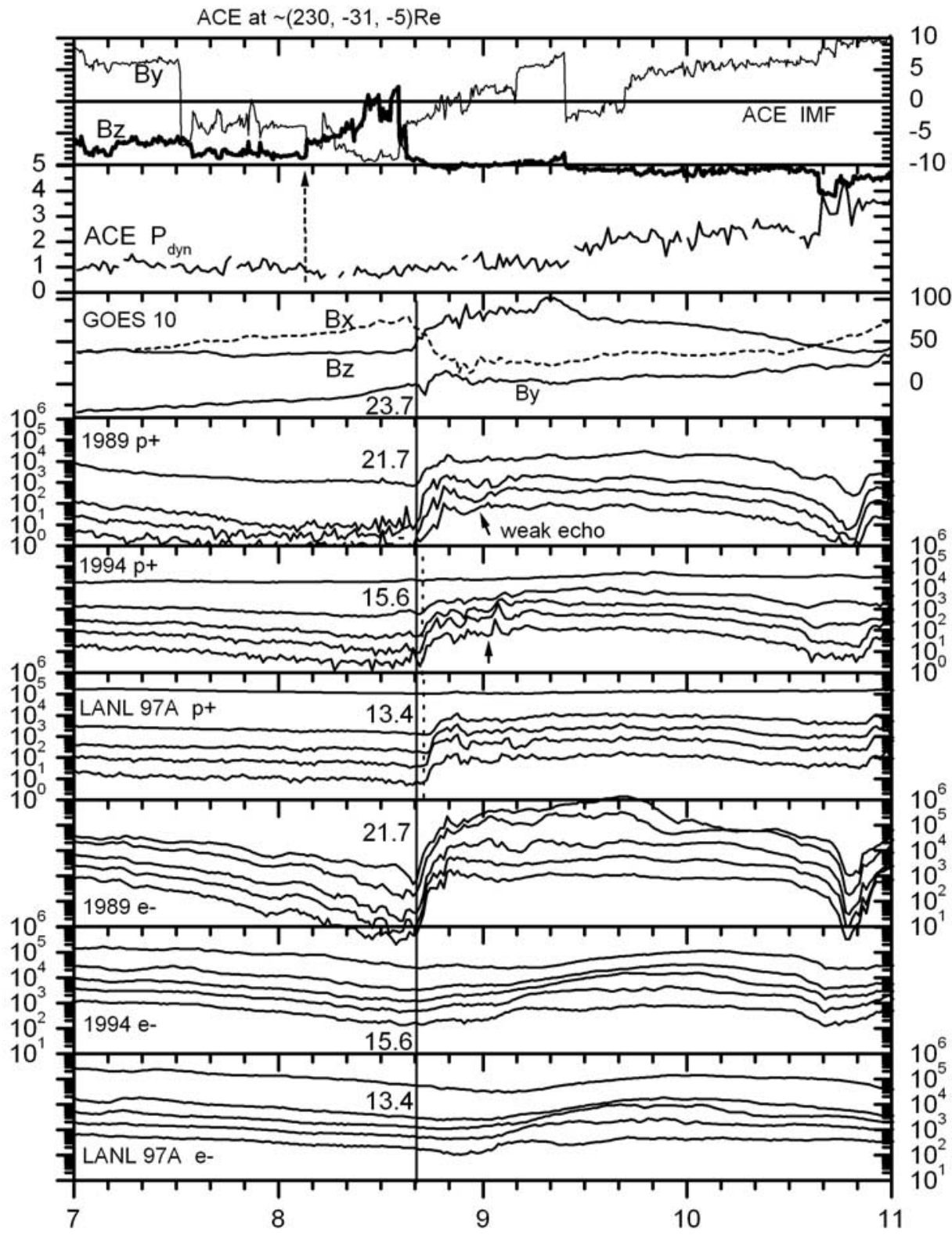

Sept 30, 2000

Figure 6. IMF $B_{y}, B_{z}$, and $P_{\text {dyn }}$ as observed by the ACE spacecraft (upper two panels), magnetic field in GSM from the GOES 10 geosynchronous spacecraft (third panel), and flux of energetic protons and electrons from the several LANL geosynchronous spacecraft for a substorm event on 30 September 2000.

strongly southward, around $-15 \mathrm{nT}$, for a prolonged period, and the dynamic pressure shows a weak increase by $\sim 30 \%$ at ACE at $\sim(226,-34,-3.3)$ Re. However, the low-latitude $\mathrm{H}$ data shows no evidence for the impact of a significant pressure enhancement. This is demonstrated in Figure 8 using the $\mathrm{H}$ data from six low-latitude stations around the Earth. The names and geographic latitude/longitude of each station are indicated in each panel. The only feature that is evident is the $\mathrm{H}$ enhancement on the nightside that is presumably due to the current wedge formation, and there is no global $\mathrm{H}$ increase that would be expected from the impact of a pressure pulse, even at stations near noon. The dipolarization seen by GOES 10 near midnight is typical, as shown in the third panel in Figure 7 . The fluxes of both species show dispersionless increases at 21.8 MLT. Then, at 15.7 MLT, the slightly delayed but still dispersionless increase of the proton fluxes is seen at least at the three highest energy channels, implying the penetration of the injection front into the afternoon side region. On the other hand, the electron flux at 15.7 MLT initially shows a sharp and brief decrease, which then recovers to a flux level that does not exceed the preonset level. At 9.3, the longerdelayed and dispersed flux increase of both species is clearly seen. For this event, global images from the WIC 


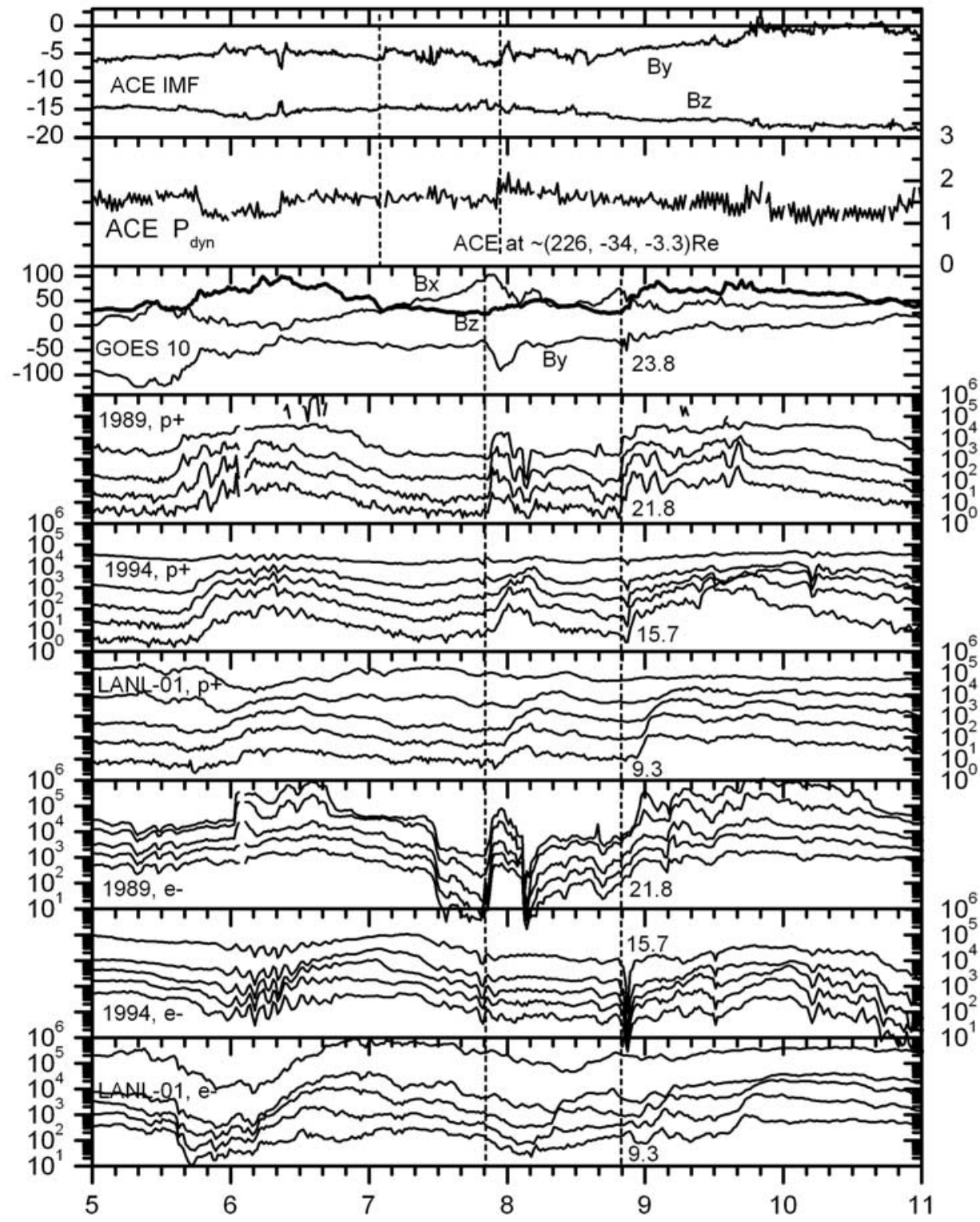

Mar 20, 2001

Figure 7. IMF $B_{y}, B_{z}$, and $P_{\mathrm{dyn}}$ as observed by the ACE spacecraft (upper two panels), magnetic field in GSM from the GOES 10 geosynchronous spacecraft (third panel), and flux of energetic protons and electrons from the several LANL geosynchronous spacecraft for a substorm event on 20 March 2001.

imager on the IMAGE spacecraft are available and indicate that the auroral surge formed over a broader MLT range than for typical substorms, which is consistent with what was demonstrated in Paper 1.

[25] In Figure 9, the substorm of interest occurred at 1618 UT. Again this substorm is associated with a strongly southward IMF, $B_{z}$ below $-10 \mathrm{nT}$ as measured by the Geotail spacecraft as shown in the top panel. The dynamic pressure at Geotail, being located at $\sim(15.6,22.4$, 4.6) $R_{E}$, shows some weak to moderate variations, but the GOES 8 being located near noon shows no evidence for the impact of a significant pressure variation at the time of substorm onset. This is further confirmed by low-latitude $\mathrm{H}$ data shown in Figure 10 in the same format as in Figure 8, which shows no evidence of the impact of a pressure increase or decrease. The dispersionless increase of the flux of both species at 23.2 MLT is clear. At 16.8 MLT, the proton flux still shows the dispersionless increase but is delayed by a few minutes. A careful interpretation of the electron flux in the last panel shows that, at this same afternoon sector 16.8 MLT, the electron flux first exhibits a weak decrease, which is promptly followed by a dispersionless increase as indicated by thick arrow. This electron flux increase is simultaneous with the proton flux increase shown in the sixth panel (dotted line), although the electron flux increase is very weak, which is then followed by highly dispersed flux enhancements as expected from injected electrons drifting around from the dawnside. This example 


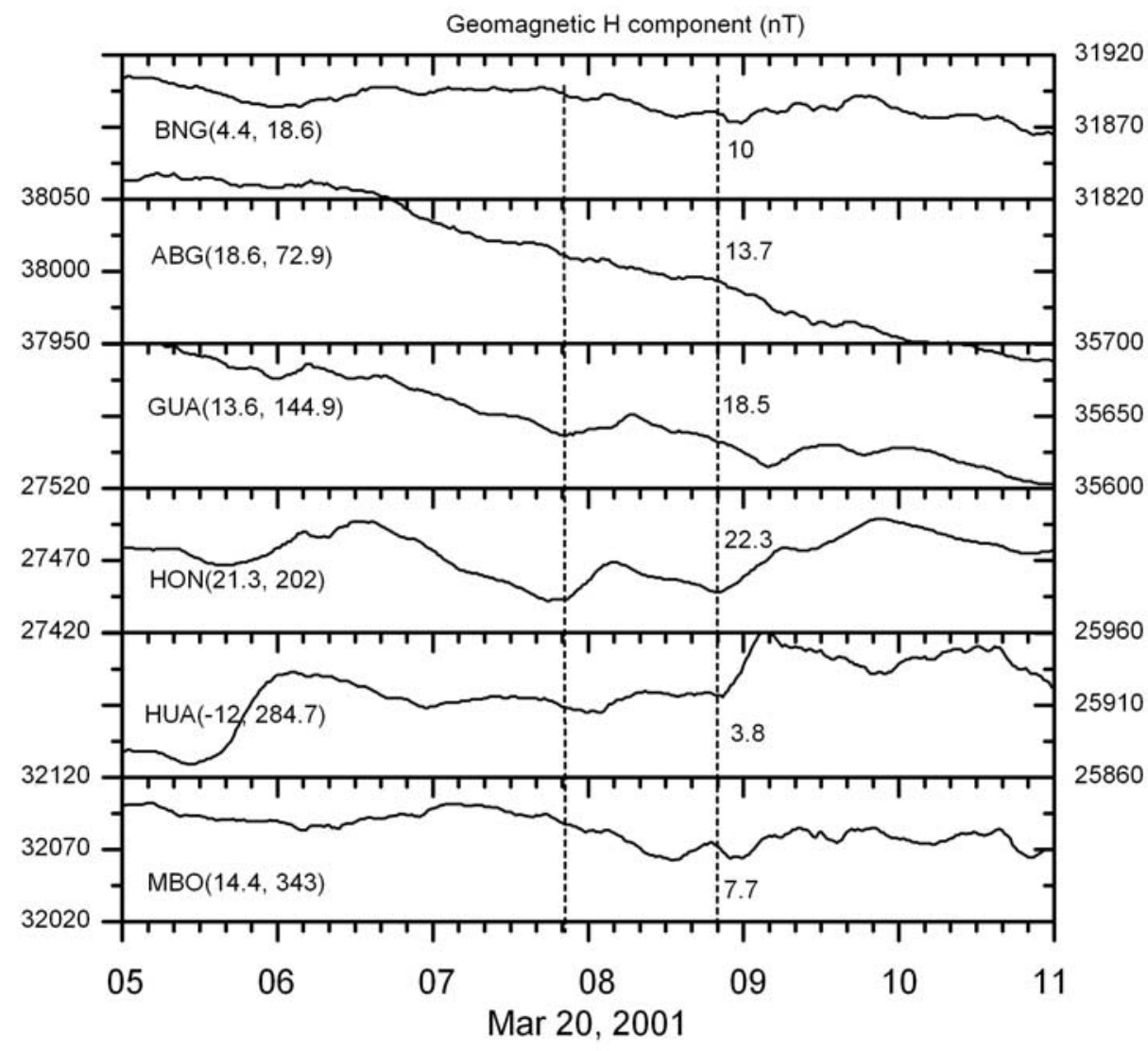

Figure 8. H-component (in $\mathrm{nT}$ ) from six low-latitude ground stations distributed in longitude around the Earth for the same substorm event as in Figure 7. The times of onset are indicated by vertical lines, and the MLT values of each station's location at $\sim 0850$ UT are given by the vertical line.

serves as a good evidence for the propagation of the injection front of both species into the afternoon sector. It is also interesting to see that the dispersionless flux increase of both species is also seen at dawn, 2.6 MLT, though it is quite delayed.

[26] In summary, substorms triggered by IMF turnings following prolonged strongly southward IMF lead to particle flux disturbances that are very similar to those for not strongly southward IMF conditions, except that dispersionless injection occurs over a significantly wider MLT region. This might relate to the broader MLT range of auroral surge formation for such events seen in Paper 1. In the examples presented here, dispersionless injection was seen as close to noon as $\sim 15.6$ MLT on the afternoonside. However, this afternoonside injection was always delayed by a few minutes, implying a propagation effect. Also this is more easily seen for protons than for electrons. In one event, we observed the dispersionless injections of both species on the dawnside as well.

\subsection{Dynamic Pressure Disturbances}

[27] Here we examine the particle flux response to a dynamic pressure enhancement in the case where the associated IMF $B_{z}$ is strongly and continuously southward for at least $30 \mathrm{~min}$ before, at the time of, and for some time after, the pressure enhancement. Also, it is required that the IMF $B_{z}$ remains approximately steady at and around the time of the pressure enhancement so that we can focus on the pure pressure effect and avoid the possible mixture of effects due to an IMF change. However, many of the pressure changes are accompanied with simultaneous changes in the IMF. This greatly limits the number of events suitable for the analysis, and so we will be content with case studies without pursuing statistical analysis. We find that as the IMF becomes strongly southward, the flux response becomes different from and more complex than the response for northward or weakly southward IMF. It also exhibits important similarities with and differences from substorm injections.

[28] The first event is presented in Figure 11 where the dynamic pressure enhances by about $100 \%$ (second panel) while the IMF $B_{z}$ is strongly southward, $\sim-11$ to $-12 \mathrm{nT}$ (top panel). An interesting feature in this event is that the pressure enhancement is in a form of three successive enhancements. This feature is well reflected in the geosynchronous magnetic field response that appears as three successive dipolarizations (third panel showing the magnetic elevation angle theta measured by two GOES spacecraft). It is also confirmed in the geomagnetic $\mathrm{H}$ response (data shown in the paper by Lee et al. [2004]). This direct compression effect is also reflected in the proton flux response near 17.3 MLT (fourth panel of Figure 11), where three successive enhancements that correspond to the three pressure enhancements can be seen at high energies. 


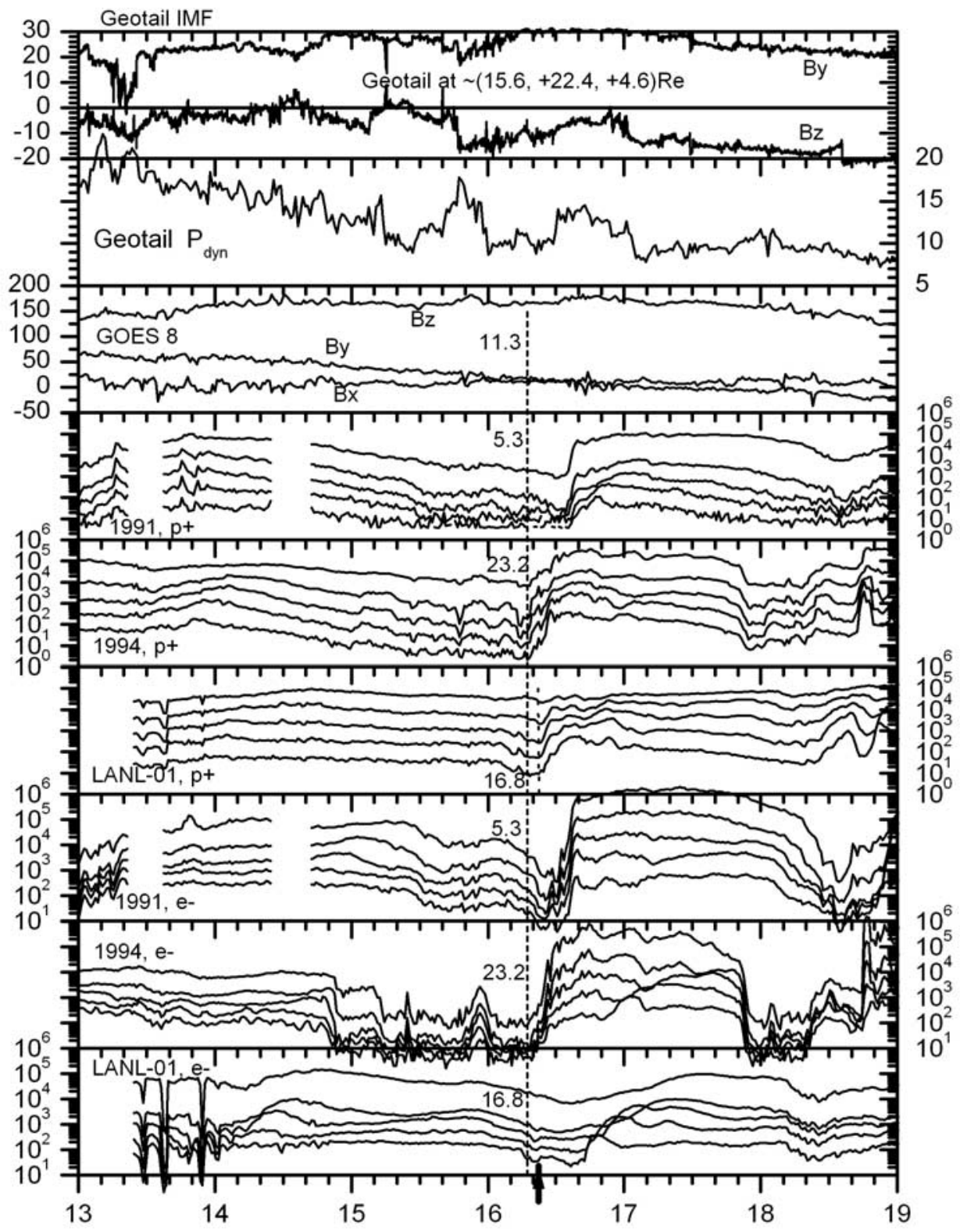

Aug 17, 2001

Figure 9. IMF $B_{y}, B_{z}$, and $P_{\mathrm{dyn}}$ as observed by the Geotail spacecraft (upper two panels), magnetic field in GSM from the GOES 8 geosynchronous spacecraft (third panel), and flux of energetic protons and electrons from the several LANL geosynchronous spacecraft for a substorm event on 17 August 2001.

That this proton flux enhancement at 17.3 MLT initiates nearly simultaneously with the initiation of the dipolarizations observed at 23.3 and 19.3 MLTs (third panel) implies that this is the global direct response to the pressure pulse impact. Note that the proton flux enhancement at 17.3 MLT is dispersionless in energy. Simultaneous with this, however, the proton fluxes at 11.2 and 9 MLTs show little change, only very weak decreases at high energies, before showing a delayed large flux increase with dispersion. This little change or weak decrease is similar to the compression effect that we have found to occasionally occur for typical pressure events associated with northward or weakly south- ward IMF. In the delayed and dispersed proton flux enhancements at 11.2 and 9 MLTs, it is seen that the time delay and the energy dispersion increase in the westward direction, namely, from 11.2 to 9 MLT, as marked by dotted lines. These features are just like what we normally see from a substorm injection. On the other hand, the electron flux initially shows dispersionless decreases at most energies and simultaneously at all three MLTs, being caused by the direct compression from the enhanced pressure impact. Note that the electron flux level then recovers and eventually increases with dispersion that increases in the eastward direction as marked by dotted lines, which is also like a 


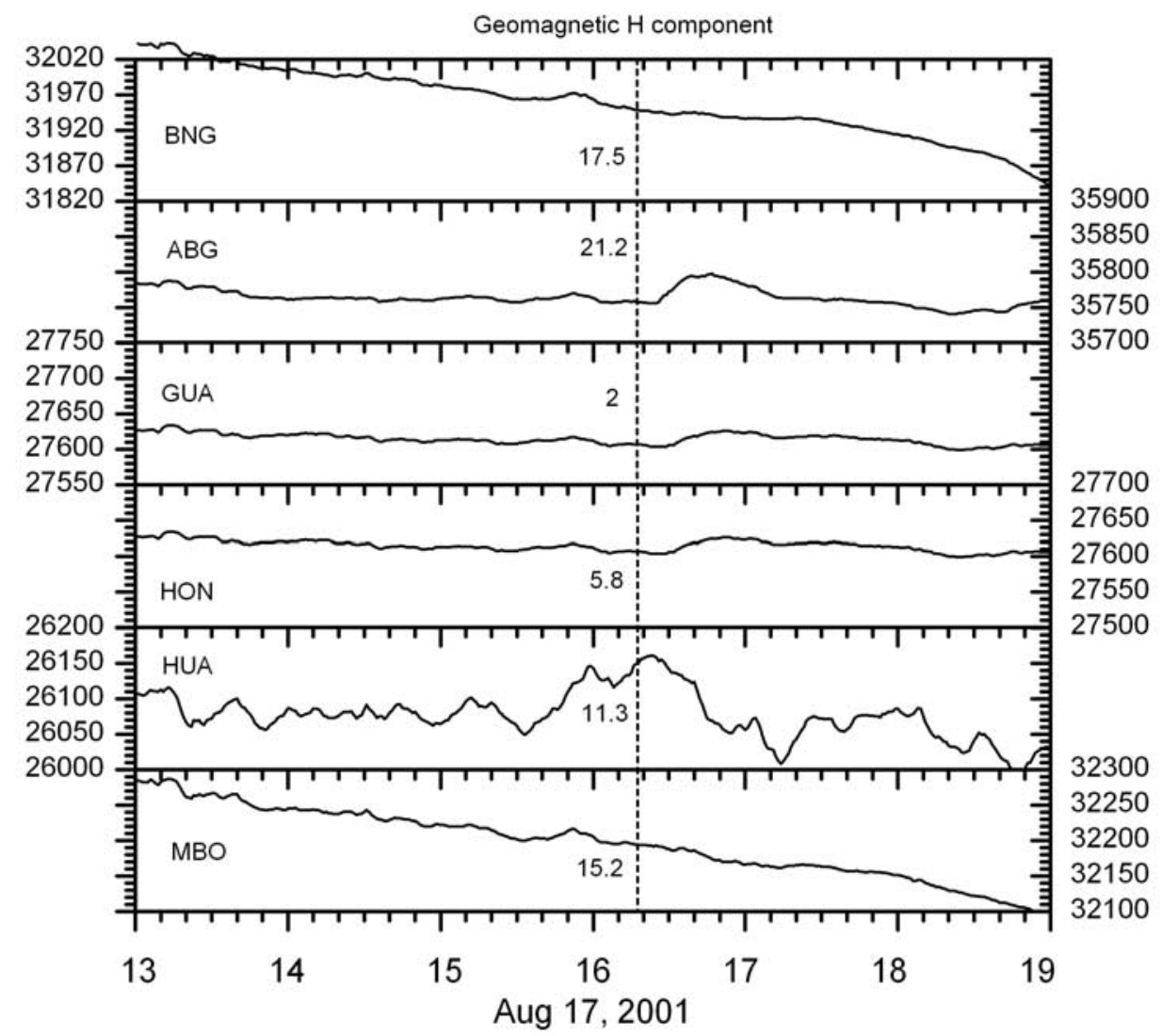

Figure 10. H-component (in $\mathrm{nT}$ ) from six low-latitude ground stations distributed in longitude around the Earth for the same substorm event as in Figure 9.

substorm-like injection. This example suggests that the dynamic pressure enhancement under the strongly southward IMF can lead to a substorm-like particle injection as well as the direct compression effect. This is clearly distinguished from the earlier three cases, namely, the typical substorm injection for not strongly southward IMF conditions, the typical $P_{\mathrm{dyn}}$ enhancement event for not strongly southward IMF conditions, and the substorm injection triggered by IMF turnings following prolonged strongly southward IMF. For these three cases, only either the direct compressional response or the substorm injection was observed, but not both. The two-mode type disturbance in aurora for this same event has been demonstrated using IMAGE WIC data in Paper 1.

[29] Further events that support the picture above are presented in the subsequent figures. Figure 12 shows an event that occurred on 4 October 2000. The 4 October 2000 storm has been previously studied by Reeves et al. [2004] and C:son Brandt et al. [2004] in association with stormsubstorm relationships. The time of interest here is indicated by vertical line. The IMF and solar wind $P_{\mathrm{dyn}}$ from ACE and Geotail are shown together in the top two panels without a time shift. The IMF $B_{z}$ is again strongly southward below $-10 \mathrm{nT}$. The LANL flux disturbance begins at $\sim 1355$ UT. The responsible pressure enhancement observed at ACE and Geotail is indicated by arrows in the second panel, but the signatures are not unambiguous. The transit time from ACE to Geotail was estimated based on the same
IMF structure being seen at both spacecraft, i.e., the abrupt further southward turning of IMF $B_{z}$ at $\sim 1335$ UT at ACE and at $\sim 1417$ UT at Geotail. The impact of the enhanced pressure is confirmed with the low-latitude $\mathrm{H}$ data as displayed in Figure 13 in the same format as in Figure 8. The global near-simultaneous increase in $\mathrm{H}$ is clearly seen at $\sim 1355$ UT. Notice that the different vertical scales were used for MBO and HUA to show $\mathrm{H}$ increases more clearly on the dayside. The geosynchronous magnetic field $B_{z}$ also indicates increases in response to this pressure enhancement as measured on the morningside and the dawnside by two GOES spacecraft (third panel of Figure 12). The particle flux response here again shows the two-mode type response, namely, the substorm-like injection plus the typical compression effect. First, the proton flux increase at 14.5 MLT reflects the direct compression effect. However it is interesting that the flux increase is not quite dispersionless, being slightly delayed at the two highest energies. (Note that this weak dispersion is in the opposite sense to what we normally see from the drift effect of the substorminjected particles.) This is likely due to the radial profile of the particle distribution at constant adiabatic invariants being different at different energies. The compression effect for the electron flux at 14.5 MLT appears as the dispersionless, though weak, decrease (before its long-delayed and dispersed increase). On the other hand, the substorm-like injection signature is clearly seen on the nightside (20.8 and 2.9 MLTs) as dispersionless increases of both species. One 


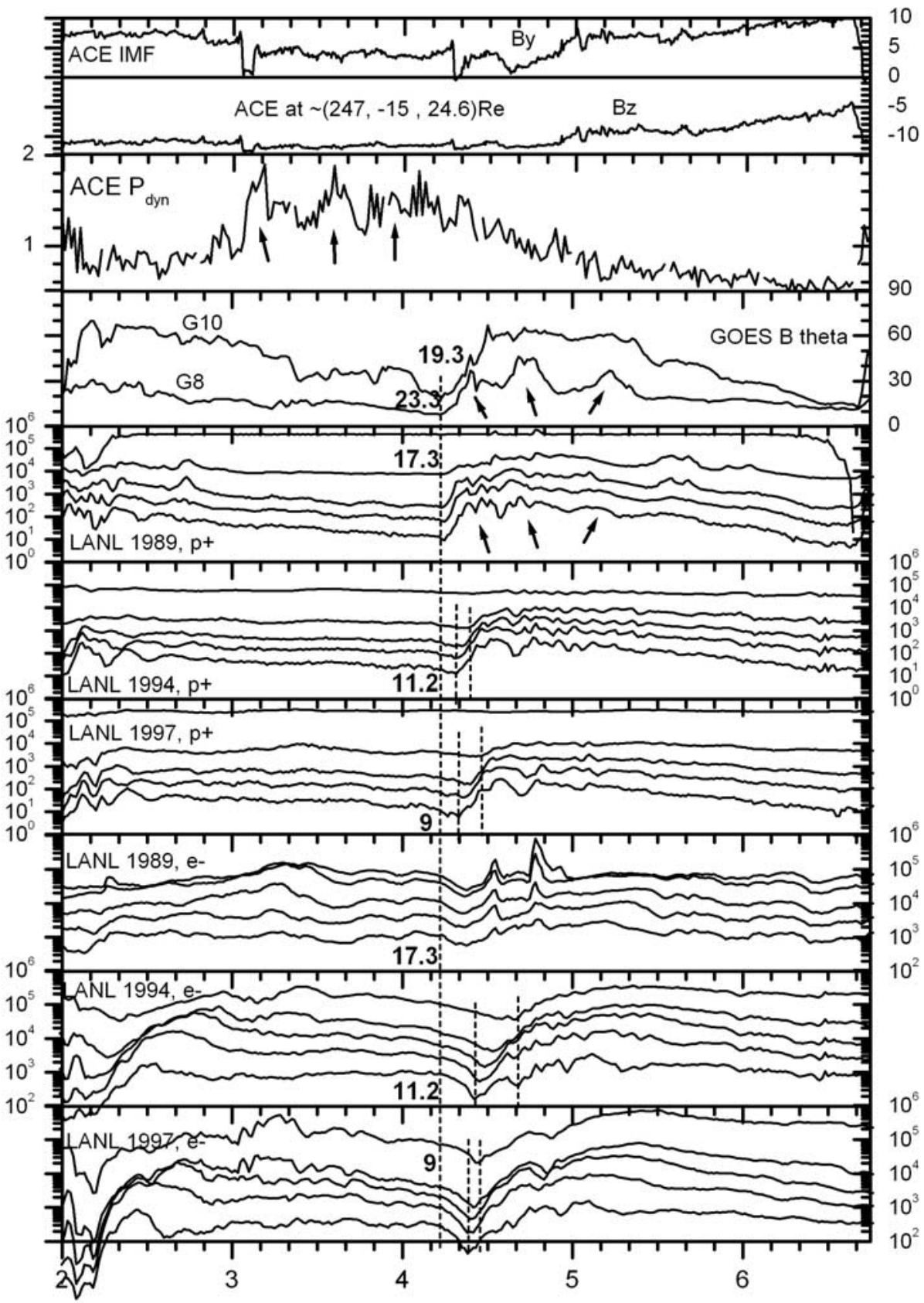

Aug 11,2000

Figure 11. IMF $B_{y}, B_{z}$, and $P_{\mathrm{dyn}}$ as observed by the ACE spacecraft (upper two panels), magnetic field elevation angle from the GOES 8 and 10 geosynchronous spacecraft (third panel), and flux of energetic protons and electrons from the several LANL geosynchronous spacecraft for a $P_{\text {dyn }}$ enhancement event on 11 August 2000.

interesting feature is that the dispersed injection near 14.5 MLT that results from the dispersionless nightside injection of particles into lower radial distances is clear in the electron fluxes, but is difficult to see in the proton fluxes. We find that this is often the case in other events that are not presented here. We think that it is a matter of balance between the compression effect (given the radial profile of the source particle distribution) and the substorm-like injection that determines the appearance of the dispersion and the drift echo. In the case of the proton, the flux disturbance due to the pressure compression seems to dominate over the disturbance due to the substorm-like injection, and it is the other way around for the electron case. Also it is interesting and perhaps not surprising that the dispersionless flux increase on the dayside, the direct compression effect near 14.5 MLT, occurs earlier than that on the nightside, the substorm injection near 20.8 MLT. 


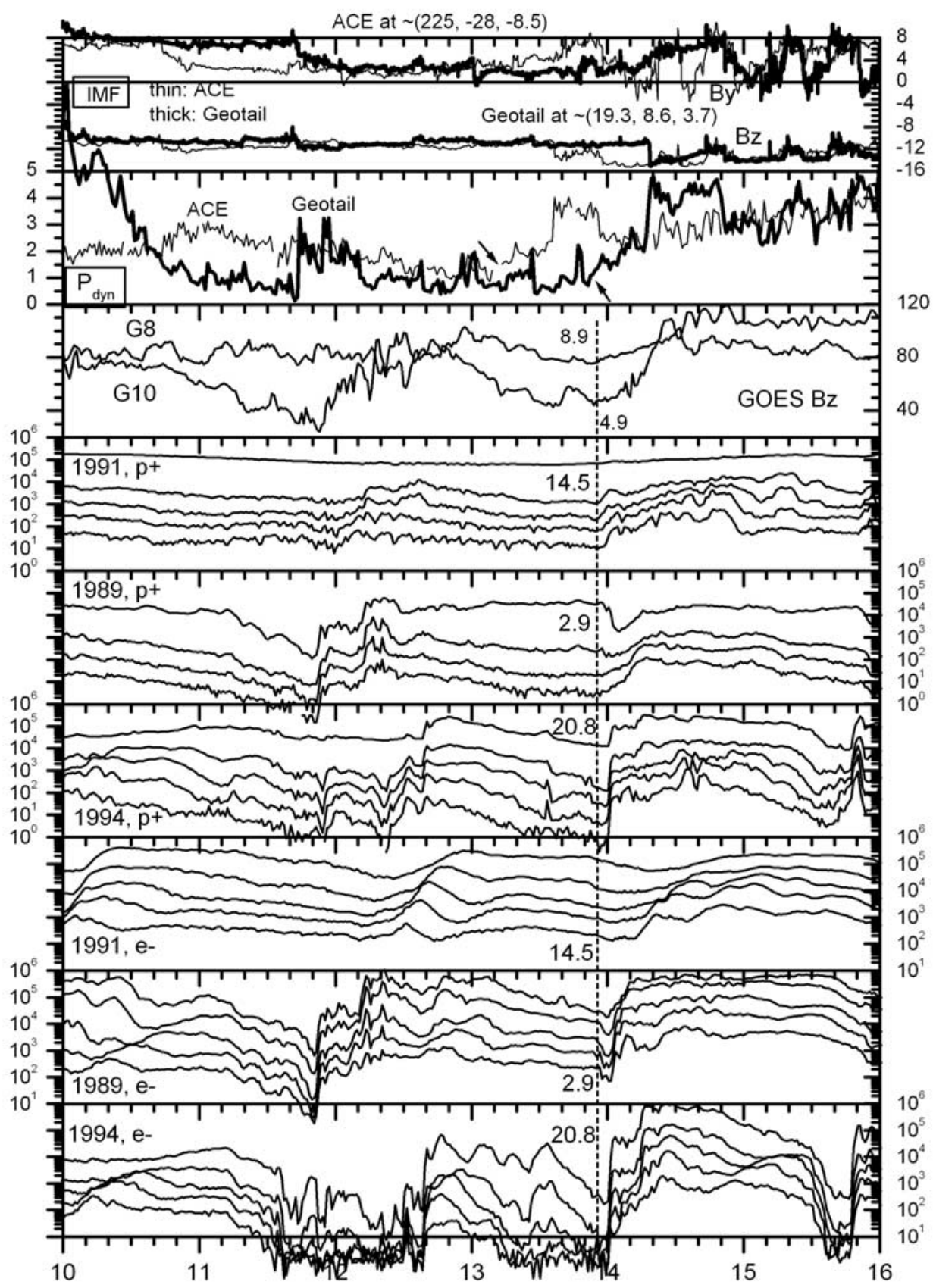

Oct 4,2000

Figure 12. IMF $B_{y}, B_{z}$, and $P_{\text {dyn }}$ as observed by the ACE and Geotail spacecraft (upper two panels), magnetic field in GSM from the GOES 8 and 10 geosynchronous spacecraft (third panel), and flux of energetic protons and electrons from the several LANL geosynchronous spacecraft for a $P_{\text {dyn }}$ enhancement event on 4 October 2000.

[30] Figure 14 shows another event that occurred on 14 October 2000. Two pressure-induced LANL flux disturbance events are identified as indicated by vertical lines. Both are associated with strongly southward IMF and $P_{\text {dyn }}$ enhancement as measured by Geotail (top two panels). For both events, the geosynchronous magnetic field dipolarization on the nightside is clearly seen through the increase in the magnetic elevation angle theta in the third panel.
The $P_{\mathrm{dyn}}$ enhancement is less clear at Geotail for the first event near 0658 UT, which is most likely due to solar wind inhomogeneity. However, the $P_{\text {dyn }}$ enhancement impact for both events is again confirmed by checking the global $\mathrm{H}$-increase at low-latitude stations (not shown). Let us consider first the flux response event that occurred near 0658 UT. At this time, three LANL spacecraft were located at 20, 13.9, and 11.7 MLTs. The substorm-like 


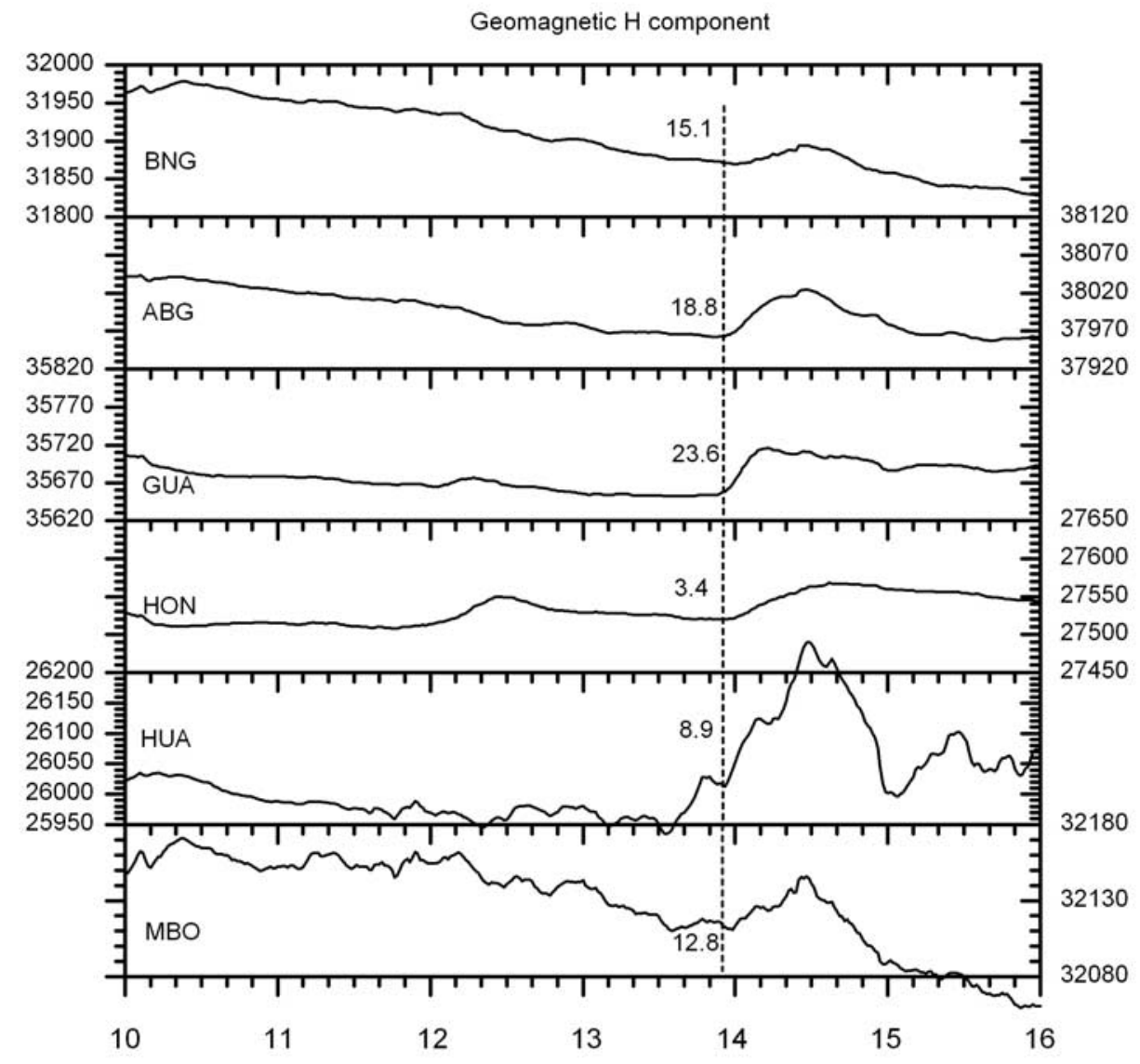

Oct 4, 2000

Figure 13. H-component (in $\mathrm{nT}$ ) from six low-latitude ground stations distributed in longitude around the Earth for the same $P_{\text {dyn }}$ enhancement event as in Figure 13.

injection on the nightside is clear through the dispersionless flux increase of both species near 20 MLT that initiates at $\sim 0705$ UT. The resulting drift effect is well seen through the delayed dispersions, particularly in the electron flux, on the dayside. The direct compression effect seems to appear most obvious in the dispersionless increase of the proton flux at 13.9 MLT. The proton flux at 11.7 MLT also shows significant increase. It is, however, rather striking to see that the proton flux increase at 11.7 MLT is actually, though weakly, dispersed in energy! This may be due to radial profiles of source particle distribution that are different for different energies. However, this feature requires further study using more events for a solid conclusion. It should however be noted that the dispersionless proton flux increase at 13.9 MLT precedes the substorm injection near 20 MLT by several minutes, implying that the disturbance at 13.9 MLT is the direct response to the $P_{\mathrm{dyn}}$ enhancement and is not the result of azimuthal drift from the nightside substorm-injection front. The direct compression effect is less clear in the electron fluxes, which show only very weak dispersionless decreases or virtually no changes at the dayside MLTs, 13.9 and 11.7, prior to the long-delayed dispersion due to the electrons injected on the nightside.
[31] Next, the second event near 0952 UT also confirms the substorm-like injection as well as the dayside direct compression effect under the strongly southward IMF, despite the data gap in one of the spacecraft. Note that the electrons at 16.7 MLT show both the dispersionless compressional enhancement at $\sim 0955$ UT and the dispersed electrons from the nightside injection (first seen in the highest energy channel initiating at 1010 to $1020 \mathrm{UT}$ ). The two-mode type of disturbance in the aurora for this same event has been demonstrated using IMAGE WIC data in Paper 1.

[32] Figure 15 shows another example of the $P_{\mathrm{dyn}}$ enhancement event under a strongly southward IMF condition. The top two panels show IMF and the dynamic pressure data from Geotail. The event of interest is marked by vertical line near 1427 UT. The $P_{\text {dyn }}$ enhancement impact is again confirmed by the geomagnetic $\mathrm{H}$ variation as shown in Figure 16. (Here we only display data from three representative stations for simplicity.) Note that the $\mathrm{H}$ curves at and after $\sim 1427$ UT in Figure 16 trace the corresponding pressure curve in the second panel in Figure 15 remarkably well. Geotail data shows several pressure variations and the $\mathrm{H}$ component tells the impact time of the first significant one. Here again the two-mode 


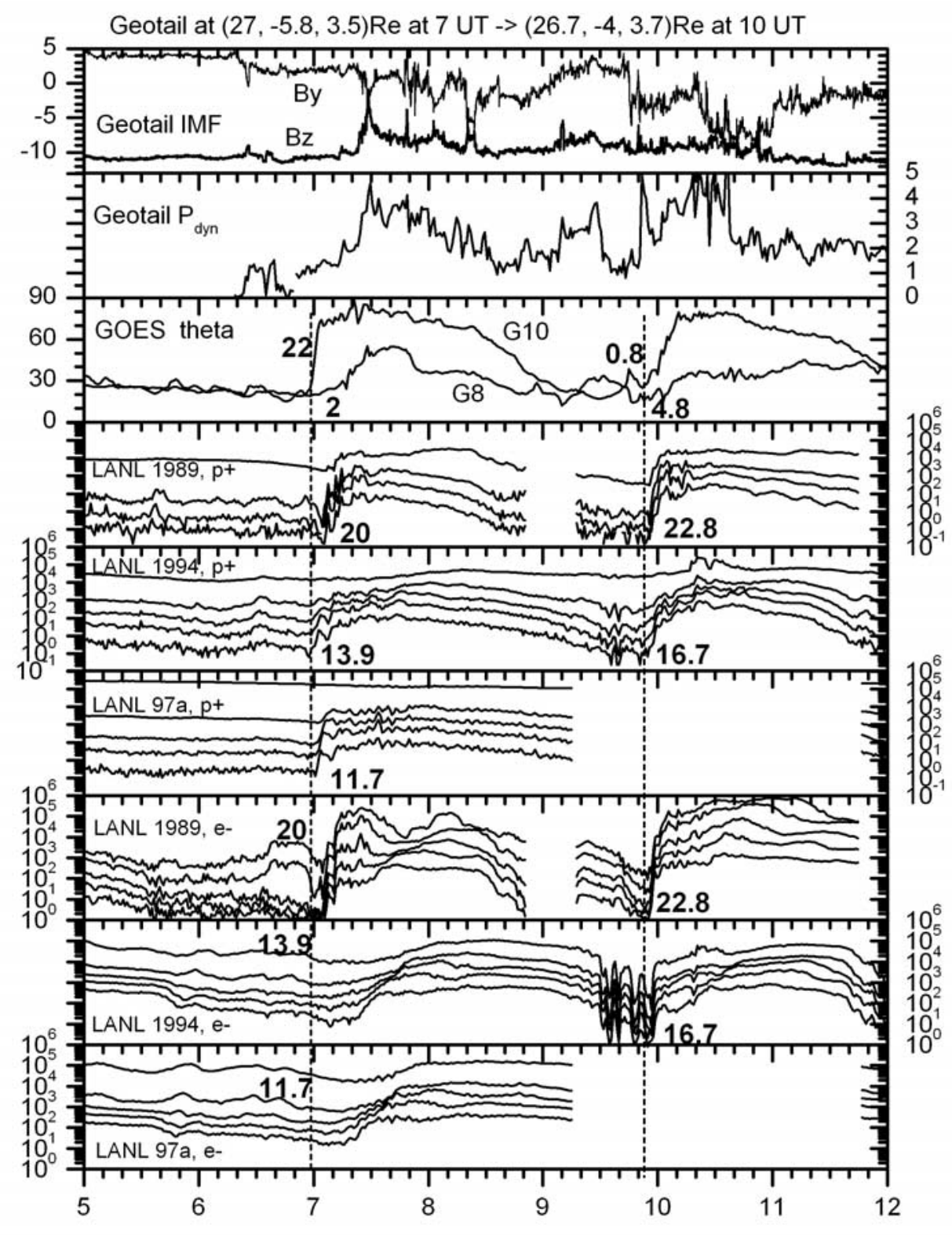

Oct 14,2000

Figure 14. IMF $B_{y}, B_{z}$, and $P_{\mathrm{dyn}}$ as observed by Geotail spacecraft (upper two panels), magnetic field elevation angle from the GOES 8 and 10 geosynchronous spacecraft (third panel), and flux of energetic protons and electrons from the several LANL geosynchronous spacecraft for $P_{\text {dyn }}$ enhancement events on 14 October 2000.

type response of the flux is well seen in the rest of the panels in Figure 15. First, the compression effect is clear on the dayside and reflected through the dispersionless increase of the proton flux at 15 MLT. Further, this dispersionless increase of the proton flux precedes those on the nightside by a few minutes. The dispersed proton flux increase near 3.5 MLT on the other hand suggests there was a true substorm-like injection on the nightside induced by the $P_{\text {dyn }}$ enhancement impact. The substorm-like injection is also evident in the electron flux disturbance in terms of nightside dispersionless increase and the associated dispersions that are most clearly seen near 15 MLT. Near 1427 UT, there is a small data gap (for about $2 \mathrm{~min}$ ) that makes it difficult to precisely determine the electron flux behavior at the time of the pressure enhancement at 15 and 12 MLT. However, a dispersionless (to within $2 \mathrm{~min}$ ) flux reduction is clear at 12 MLT.

[33] Summarizing the result here briefly, a $P_{\text {dyn }}$ enhancement under strongly southward IMF results in a substormlike injection as well as the direct compression effect. The dispersed drift injections away from the nightside that reflect the substorm injection is present in both species response but is clearer in the electron fluxes. The compression effect is often more evident in the proton flux response. 


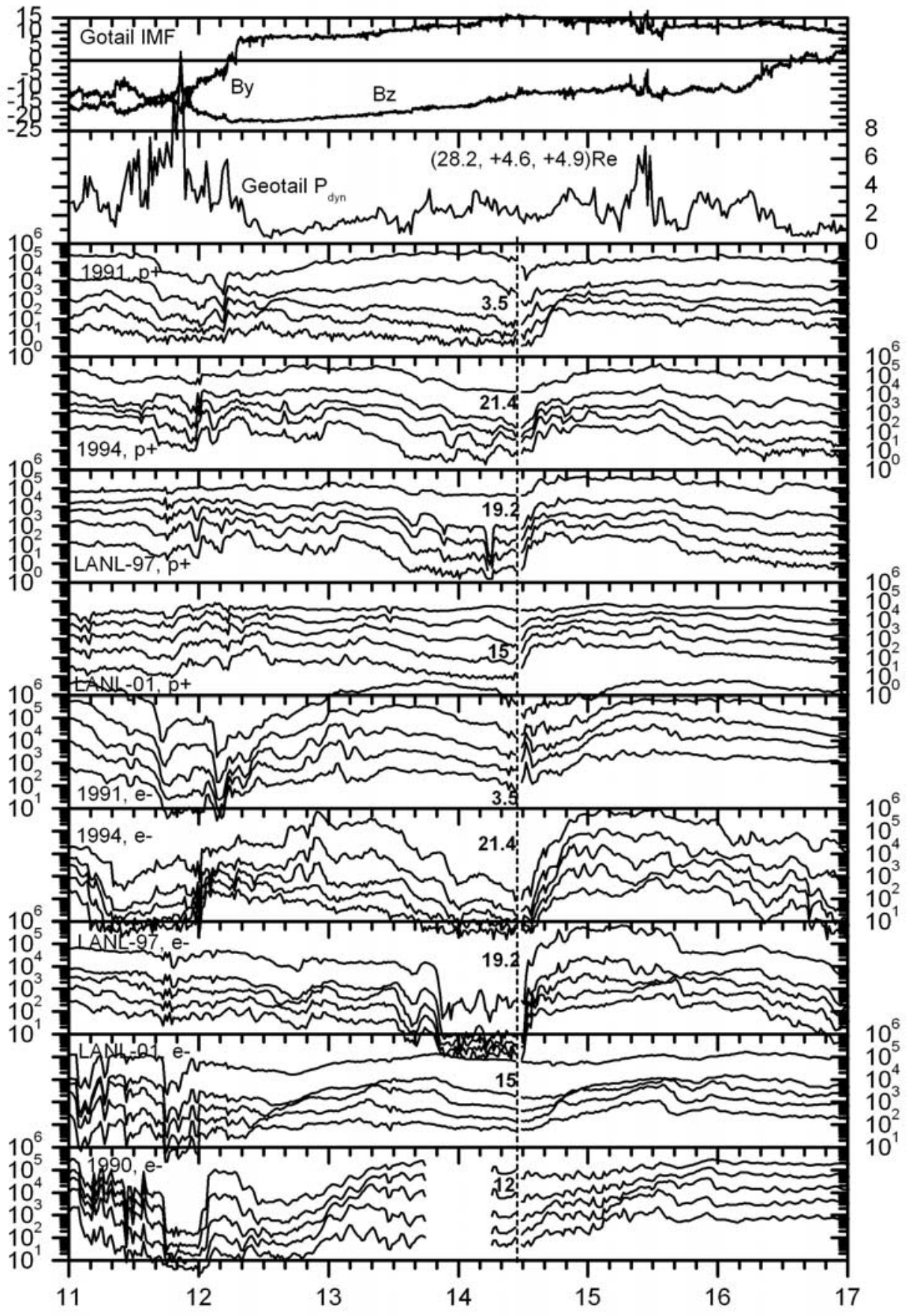

Oct 3, 2001

Figure 15. IMF $B_{y}, B_{z}$, and $P_{\mathrm{dyn}}$ as observed by Geotail spacecraft (upper two panels), and flux of energetic protons and electrons from the several LANL geosynchronous spacecraft for a $P_{\text {dyn }}$ enhancement event on 3 October 2001.

Also, not surprisingly, the compression effect on the dayside precedes the substorm-like injection, at least, in the later three events presented in this subsection.

\section{Discussion and Summary}

[34] In summary, we have presented the results of analysis of the geosynchronous energetic particle flux disturbances caused by substorms and dynamic pressure enhancements. Four types of disturbances were considered separately and compared with each other: substorm injections caused by sole IMF changes for not strongly southward IMF conditions, dynamic pressure enhancement events for not strongly southward IMF conditions, substorm injections triggered by IMF turnings following prolonged strongly southward IMF but associated with 


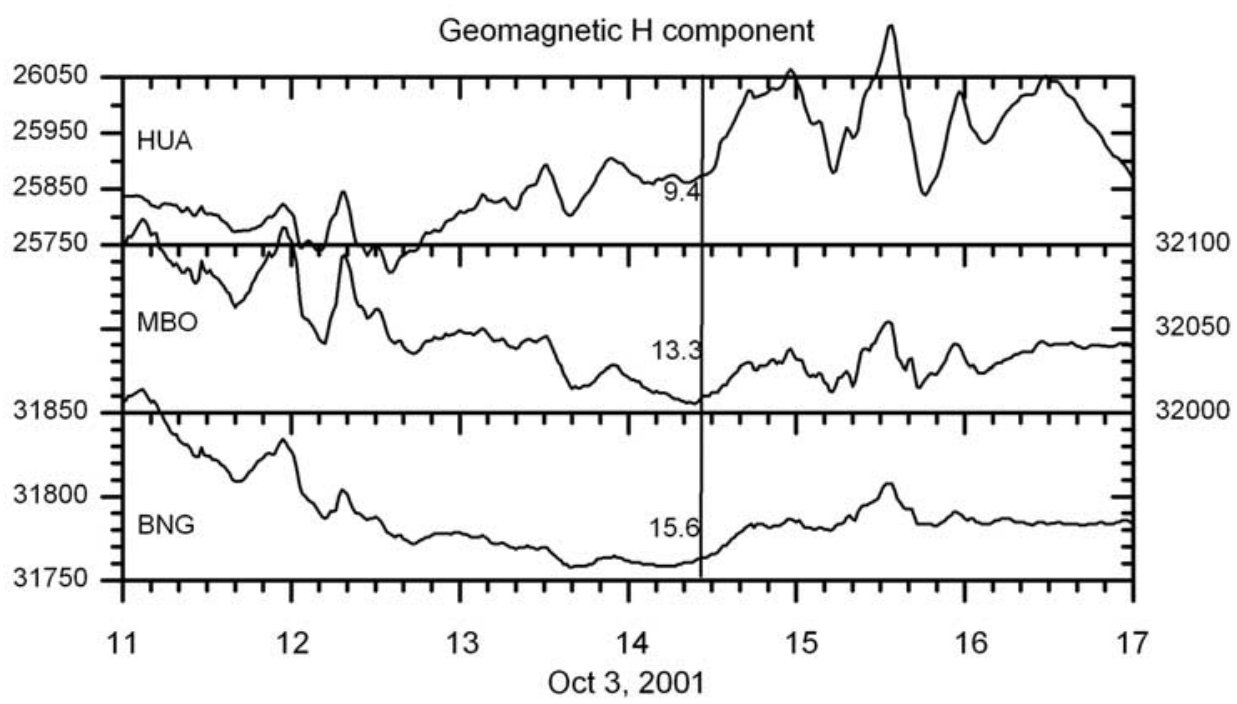

Figure 16. H-component (in $\mathrm{nT}$ ) from three representative dayside low-latitude ground stations for the same $P_{\text {dyn }}$ enhancement event as in Figure 15.

no dynamic pressure enhancement, and dynamic pressure enhancement events under steady and strongly southward IMF conditions. We have found that the geosynchronous particle flux disturbances are clearly distinguished among these four types.

[35] The key features of typical substorm injection under not strongly southward IMF conditions are well-known: the nightside injection zone and the dispersed flux increase elsewhere. The dynamic pressure enhancement under not strongly IMF conditions leads to the flux increase or decrease with no dispersion in energy or sometimes no change at all, depending on the radial profile of the source particle distribution at constant first and second adiabatic invariants. We see no evidence for substorm-like injection. There is only the simple magnetospheric compression effect, which is consistent with the result using the auroral image data in Paper 1. In this case we also find that the response propagates from the dayside toward the nightside.

[36] For substorms triggered by sole IMF turnings following prolonged strongly southward IMF, the substorm injection feature is very similar to that for less strongly southward IMF condition, except that the injection front becomes wider in MLT coverage. The dispersionless flux increase is seen even on the afternoonside mostly for protons. Also it is interesting to note that as shown in Paper 1, the MLT coverage by the substorm auroral surge under the same IMF condition is much less wide than that covered by the proton injection front.

[37] On the other hand, dynamic pressure enhancements under a strongly southward IMF condition lead to a twomode response. The geosynchronous particles show the substorm-like injection signature associated with current wedge formation on the nightside as well as the simple compression effect primarily on the dayside. The compression effect is in fact the same as that for not strongly southward IMF conditions. Also the dayside compressioninduced disturbance occurs earlier by a few minutes than the substorm-like injection on the nightside. All these flux disturbance features here are consistent with the two-mode response seen in the auroral disturbance studied using the IMAGE WIC data in Paper 1.

[38] Last, Paper 1 presents several null events where the interplay between an IMF turning and a simultaneous dynamic pressure change leads to no auroral activity. In reality, it is quite common that the solar wind exhibits changes in both the dynamic pressure and the IMF, often simultaneously. The interplay between the two is of interest particularly when the accompanied IMF is southward and warrants a systematic study. In this Paper 2, we have not studied the geosynchronous particle flux disturbances for such events, leaving it as a future work.

[39] Our results are closely related to particle flux disturbances during magnetic storms. IMF turnings and dynamic pressure enhancements often exist during magnetic storm period and can be a direct cause of the observed flux disturbances. In particular, strong magnetic storms are associated with strongly southward IMF conditions. This is exactly the situation that we have been primarily concerned about in the present work. The geosynchronous particle flux disturbances during magnetic storms can therefore be understood based on the present results. Some magnetic storms are associated with sawtooth oscillations where continuous quasi-periodic particle flux disturbances occur quite globally [e.g., C:son Brandt et al., 2004; Lee et al., 2004; Lui et al., 2004; Reeves et al., 2004]. In fact, the dynamic pressure events for strongly southward IMF conditions in section 4.2 are sampled from sawtooth oscillation events. Our results here suggest that the two-mode response to a dynamic pressure under strongly southward IMF conditions can be a likely explanation for the global nature of many of the sawtooth flux disturbances.

[40] Acknowledgments. This work was supported by grant R012002-000-00100-0 from Basic Research Program of the Korea Science and Engineering Foundation. This research was supported at UCLA in part by NSF grants OPP-0136139 and ATM-0207298. The energetic particle 
flux data were obtained from the Los Alamos National Laboratory geosynchronous spacecraft. The GOES magnetic field data were provided by H. Singer through NASA's cdaweb site. The solar wind plasma and magnetic field data of ACE, WIND, and GEOTAIL used here were obtained from NASA's cdaweb site, and we are grateful to each of the principal investigators, D. J. McComas, N. Ness, K. Ogilvie, R. Lepping, L. Frank, and S. Kokubun. The geomagnetic data used were obtained from WDC at Copenhagen.

[41] Arthur Richmond thanks Renata Lukianova and another reviewer for their assistance in evaluating this paper.

\section{References}

Birn, J., et al. (1997), Characteristic plasma properties during dispersionless substorm injections at geosynchronous orbit, J. Geophys. Res., 102, 2309.

Birn, J., M. F. Thomsen, J. E. Borovsky, G. D. Reeves, and M. Hesse (2000), Particle acceleration in the dynamic magnetotail, J. Geophys. Res., 105, 2149.

Boudouridis, A., et al. (2003), The effect of solar wind pressure pulses on the size and strength of the auroral oval, J. Geophys. Res., 108(A4), 8012, doi:10.1029/2002JA009373.

Boudouridis, A., E. Zesta, L. R. Lyons, P. C. Anderson, and D. Lummerzheim (2004), Magnetospheric reconnection driven by solar wind pressure fronts, Ann. Geophys., 22, 1367.

Chua, D., G. Parks, M. Brittnacher, W. Peria, G. Germany, J. Spann, and C. Carlson (2001), Energy characteristics of auroral electron precipitation: A comparison of substorms and pressure pulse related auroral activity, J. Geophys. Res., 106, 5945.

Collier, M. R., et al. (1998), Multispacecraft observations of sudden impulses in the magnetotail caused by solar wind pressure discontinuities: WIND and IMP 8, J. Geophys. Res., 103, 17,293.

C:son Brandt, P., D. G. Micthell, and S. Ohtani, et al. (2004), Storm-substorm relationship during the 4 October, 2000 storm. IMAGE global ENA imaging results, in Disturbances in Geospace: The Storm-Substorm Relationship, Geophys. Monogr. Ser., vol. 142, edited by A. S. Sharma et al., AGU, Washington, D. C.

Fairfield, D. H., and J. Jones (1996), Variability of the tail lobe field strength, J. Geophys. Res., 101, 7785.

Kawano, H., et al. (1992), Rotational polarities of sudden impulses in the magnetotail lobe, J. Geophys. Res., 97, 17,177.

Kim, K.-H., et al. (2004), Cluster observations in the magnetotail during sudden and quasiperiodic solar wind variations, J. Geophys. Res., 109 A04219, doi:10.1029/2003JA010328.

Lee, D.-Y., and L. R. Lyons (2004), Geosynchronous magnetic field response to solar wind dynamic pressure pulse, J. Geophys. Res., 109, A04201, doi:10.1029/2003JA010076.

Lee, D.-Y., L. R. Lyons, and K. Yumoto (2004), Sawtooth oscillations directly driven by solar wind dynamic pressure enhancements, J. Geophys. Res., 109, A04202, doi:10.1029/2003JA010246.

Li, X., et al. (2003), Energetic particle injections in the inner magnetosphere as a response to an interplanetary shock, J. Atmos. Sol. Terr. Phys., 65, 233.
Lui, A. T. Y., et al. (2004), Magnetotail behavior during storm time sawtooth injections, J. Geophys. Res., 109, A10215, doi:10.1029/ 2004JA010543.

Lukianova, R. (2003), Magnetospheric response to the solar wind dynamic pressure inferred from polar cap index, J. Geophys. Res., 108(A12), 1428, doi:10.1029/2002JA009790.

Lyons, L. R., G. T. Blanchard, J. C. Samson, R. P. Lepping, T. Yamamoto, and T. Moretto (1997), Coordinated observations demonstrating external substorm triggering, J. Geophys. Res., 102, 27,039.

Lyons, L. R., et al. (2000), Auroral disturbances during the January 10, 1997 magnetic storm, Geophys. Res. Lett., 27, 3237.

Lyons, L. R., D.-Y. Lee, C.-P. Wang, S. B. Mende, and H. U. Frey (2005), Global auroral responses to abrupt solar wind changes: Dynamic pressure, substorm, and null events, J. Geophys. Res., 110, A08208, doi:10.1029/2005JA011089.

Nakai, H., Y. Kamide, and C. T. Russell (1991), Influences of solar wind parameters and geomagnetic activity on the tail lobe magnetic field: a statistical study, J. Geophys. Res., 96, 5511.

Ostapenko, A. A., and Y. P. Maltsev (1998), Three-dimensional magnetospheric response to variations in the solar wind dynamic pressure, Geophy. Res. Lett., 25, 261.

Reeves, G. D. (1998), New perspectives on substorm injections, in Substorms-4, Proceedings of International Conference on Substorms-4, Hamanako, Japan, edited by S. Kokubun and Y. Kamide, p. 785, Springer, New York.

Reeves, G. D., et al. (2004), IMAGE, POLAR, and geosynchronous observations of substorms and ring current ion injection, in Disturbances in Geospace: The Storm-Substorm Relationship, Geophys. Monogr. Ser. vol. 142, edited by A. S. Sharma et al., pp. 91-101, AGU, Washington, D. C.

Russell, C. T., et al. (1994), Sudden impulses at low-latitude stations: Steady state response for northward interplanetary magnetic field, J. Geophys. Res., 99, 253.

Wing, S., D. G. Sibeck, M. Wiltberger, and H. Singer (2002), Geosynchronous magnetic field temporal response to solar wind and IMF variations, J. Geophys. Res., 107(A8), 1222, doi:10.1029/2001JA009156.

Zesta, E., et al. (2000), The effect of the January 10, 1997, pressure pulses on the magnetosphere-ionosphere current system, in Magnetospheric Current Systems, Geophys. Monogr. Ser, vol. 118, edited by S.-I. Ohtani et al., p. 217, AGU, Washington, D. C.

D.-Y. Lee, Department of Astronomy and Space Science, College of Natural Science and Institute for Basic Science Research, Chungbuk National University, Cheongju, Chungbuk 361-763, South Korea. (daeyoung-lee@hanmail.net)

L. R. Lyons, Department of Atmospheric Sciences, University of California, Los Angeles, CA 90095-1565, USA. (larry@atmos.ucla.edu)

G. D. Reeves, Space Science and Applications, ISR-1, Mail Stop D-466, Los Alamos National Laboratory, Los Alamos, NM 37545, USA. (reeves@lanl.gov) 